\title{
SUPERCONDUCTING CRITICAL TEMPERATURE AND SOFTENING OF THE PHONON SPECTRUM IN ULTRATHIN NB AND NBN/GRAPHENE HYBRIDS
}

\author{
S. L. Prischepa, ${ }^{1,2}$ V. N. Kushnir,,${ }^{1,3}$ C. Cirillo, ${ }^{4}$ V. Granata, ${ }^{5}$ I. V. Komissarov,,${ }^{1,2}$ \\ N. G. Kovalchuk, ${ }^{1}$ M. M. Mikhalik, ${ }^{1}$ A. L. Danilyuk, ${ }^{1}$ I. A. Svito, ${ }^{3}$ \\ M. Andrulevičius, ${ }^{6}$ and C. Attanasio ${ }^{5}$
}

${ }^{1}$ Belarusian State University of Informatics and Radioelectronics, P. Browka 6, 220013 Minsk, Belarus

${ }^{2}$ National Research Nuclear University (MEPhI), Kashirskoe Highway 31, 115409 Moscow, Russia

${ }^{3}$ Belarusian State University, Nezalezhnasci av. 4, 220030 Minsk Belarus

${ }^{4}$ CNR-SPIN, c/o Università degli Studi di Salerno, I-84084 Fisciano (Sa), Italy

${ }^{5}$ Dipartimento di Fisica "E.R. Caianiello", Università degli Studi di Salerno, I-84084 Fisciano $(\mathrm{Sa})$, Italy

${ }^{6}$ Institute of Materials Science, Kaunas University of Technology, 51423 Kaunas, Lithiania

\begin{abstract}
Superconductivity is studied in hybrids consisting of ultrathin superconducting film/few layer graphene. Two different superconductors were used at this purpose, $\mathrm{Nb}$ and $\mathrm{NbN}$. An increase of the superconducting critical temperature, $T_{\mathrm{c}}$, is observed when graphene is put into contact with $\mathrm{Nb}$. The largest increase is obtained for the thinnest $\mathrm{Nb}$ layer, which has a $T_{\mathrm{c}} 8 \%$ larger with respect to the single $\mathrm{Nb}$ film. In the case of $\mathrm{NbN}$ the effect is not so pronounced. Experimental data are discussed by considering the possible modification of the phonon spectrum in the superconductor due to the presence of the graphene. Within an elementary one-dimensional model based on an elastic coupling between nearestneighbor atoms, we demonstrate that the phonon spectrum in the superconductor is modified at low energies with the subsequent enhancement of the effective electron-phonon coupling constant. While the strong oscillating nature of the electron-phonon interaction, $\alpha^{2}(\omega)$, in $\mathrm{NbN}$ could lead to the insensitivity of $T_{\mathrm{c}}$ on the low-energy phonons generated by the graphene, the almost constant behavior of $\alpha^{2}(\omega)$ in $\mathrm{Nb}$ favors the increase of the superconducting critical temperature.
\end{abstract}




\section{Introduction}

The problem of tuning of the critical temperature $T_{\mathrm{c}}$ by affecting the phonon spectrum of a superconducting material arose naturally after that Eliashberg developed the equations of the theory of superconductivity [1,2] (see also [3-8]) without limiting the value of the electron-phonon interaction (EPhI). The solution of the equations is expressed in terms of the Eliashberg spectral function $\alpha^{2}(\omega) F(\omega)$ where $\alpha^{2}(\omega)$ determines the strength of the EPhI and $F(\omega)$ represents the phonon density of states (PhDOS). By calculating the spectral function, or by extracting it from tunneling measurements [1, 9-12], and then solving the Eliashberg equations, it is possible to fully describe the superconducting properties of the metal. However, the broad application of the Eliashberg's theory to the interpretation of experimental results became possible only after the work of Macmillan [4], reanalyzed later on by Allen and Dynes [7]. Based on the approximated solution of the Eliashberg integral equations, McMillan obtained a simple formula for $T_{\mathrm{c}}$ [4],

$$
T_{C}=\frac{T_{D}}{1.45} \exp \left[-\frac{1.04(1+\lambda)}{\lambda-\mu^{*}(1+0.62 \lambda)}\right]
$$

where $\lambda$ is the effective electron-phonon coupling constant, defined as

$$
\lambda=2 \int_{0}^{\infty} \frac{\alpha^{2}(\omega) F(\omega)}{\omega} d \omega
$$

$\mu^{*}$ is the pseudopotential of the screened Coulomb interaction of electrons and $T_{D}$ is the Debye temperature. The prefactor $T_{D} / 1.45$ and numbers in the exponent of Eq. (1) were fixed by obtaining the value of $T_{\mathrm{c}}$ of a bulk $\mathrm{Nb}, T_{\mathrm{c}}=9.2 \mathrm{~K}$, for a given Eliashberg function $\left(\lambda=0.82, \mu^{*} \approx 0.13\right)$ [4]. Further, various expressions for $T_{\mathrm{c}}$, which include the moments of the spectral function, $\ln \omega_{\text {ln }}=$ $\frac{2}{\lambda} \int_{0}^{\infty} \ln \omega \alpha^{2}(\omega) F(\omega) d(\ln \omega), \quad\left\langle\omega^{n}\right\rangle=\frac{2}{\lambda} \int_{0}^{\infty} \omega^{n-1} \alpha^{2}(\omega) F(\omega) d \omega$, have been considered [10]. Within these approximations, the enhancement of the superconductivity when the disorder of the crystal structure is increased or when the thickness of a superconducting film is decreased can be quite reasonably explained as a "softening" of the phonon spectrum [7,13-15]. Indeed, it follows from Eq. (2) that an increase in the weight of low-frequency phonons implies an increase in $\lambda$. This, in turn, leads to an increase in $T_{\mathrm{c}}$, see Eq. (1). In particular, in 
the case of ultrathin superconducting films, i.e. films with a thickness of a few lattice constants, the softening of the phonon spectra obtained by depositing metal nanoparticles on the film surface has been demonstrated to cause an increase of $T_{\mathrm{c}}$ [16-19]. On the contrary, the quality of the surface in ultrathin superconducting films can have a strong detrimental effect on the values of the critical temperature, as observed and theoretically explained in proximity-coupled superconductor/normal metal (S/N) hybrids [20-24].

The Eliashberg function for $\mathrm{Nb}$, measured by tunnel spectroscopy [25] and calculated with $\lambda \approx 1.24$ and $\mu^{*} \approx 0.21$ [11] reveals that the $\mathrm{EPhI}$ is significant in the frequency range 2-7 THz. It should be noted that the precise shape of the Eliashberg function as well as the values of $\lambda$ and $\mu^{*}$ depend on the quality of the samples. In tunnel experiments, the unavoidable surface imperfections cause a weakening of the electron-phonon coupling. Moreover, samples with different quality of the surface are characterized by different values of $\lambda$ and $\mu^{*}[11,25,26]$. For example, it is well established that the presence of niobium oxides on the surface leads to a distortion of the intermediate coupling picture and $\lambda \leq 0.6$ [27$29]$. When this layer is removed, the effective electron coupling value is restored to $\lambda \approx 0.9-1.2[11,30,31]$. Therefore, if an independent source of phonons within the frequency range $2-7 \mathrm{THz}$ is available, the superconductivity in $\mathrm{Nb}$ ultrathin films could be reestablished, independently on the quality of its surface.

The increase in the $T_{\mathrm{c}}$ value due to the enhancement of the electron-phonon coupling has already been shown for $\mathrm{MgB}_{2}$ films. In this case, $T_{\mathrm{c}}$ is increased up to $41.8 \mathrm{~K}$ due to softening of the bond-stretching $E_{2 \mathrm{~g}}$ phonon mode induced by the presence of a tensile strain when a thin $\mathrm{MgB}_{2}$ film is deposited onto a SiC substrate [32]. Other substrates with biaxial lattice constant larger than in bulk $\mathrm{MgB}_{2}$, such as $\mathrm{AlN}, \mathrm{GaN}, \mathrm{AlGaN}, \mathrm{MgCaB}$, were also used to confirm the modification of the phonon spectrum in $\mathrm{MgB}_{2}$ thin films [33]. Softening of the $E_{2 \mathrm{~g}}$ mode in $\mathrm{MgB}_{2}$ was also observed when $\mathrm{MgB}_{2}$ was put into contact with graphene microsubstrates [34].

However, the phonon spectra can be also modified by putting the superconductor in contact with an external source of phonons and, for this purpose, graphene is a very promising material. In this case, the role of the surface of the superconducting film is of paramount importance. Phonon spectra of graphene were deeply investigated in conjunction with the thermal conducting properties of the material. 
Thermal conductivity of suspended single layer graphene (SLG) was measured to be as high as $3000-5300 \mathrm{~W} / \mathrm{mK}[35,36]$ and this relevant behavior is mainly due to phonons rather than electrons because of the strong covalent bond of light carbon atoms [37]. In supported graphene, due to the propagation of phonons towards the substrate, the thermal conductivity becomes lower $\left(600 \mathrm{~W} / \mathrm{mK}\right.$ on $\left.\mathrm{SiO}_{2}\right)$ but still remaining larger than in ordinary metals [37]. For this reason, graphene is considered as an excellent material for heat removal in nanoelectronics and the study of its thermal properties is the subject of intensive research [38-46].

As demonstrated by equilibrium molecular dynamic simulations and by relaxation time approximation methods, the interaction with the substrate does not affect the optical, longitudinal acoustic (LA) and transverse acoustic (TA) phonon modes of a SLG [47]. However, the out-of-plane acoustic (ZA) graphene modes change significantly due to the interaction with the substrate. Calculations have shown that several low-frequency phonon modes can occur in the $\mathrm{Cu}$ substrate in the direction perpendicular to the interface, in the frequency range 2-8 THz [47]. Because these phonons propagate into the substrate from SLG, the effect of their contribution to the in-plane thermal transport is reduced to a negligible amount. A modification of a phonon spectrum is obtained when the number of the graphene layers (few layer graphene, FLG) is increased. By applying a double resonant Raman spectroscopy, the layer breathing phonon modes were detected in a few layer suspended graphene in the low frequency range $3-7 \mathrm{THz}[48,49]$. As was shown later in Ref. [50], this low energy layer breathing mode has been observed also in supported FLG on $\mathrm{SiO}_{2} / \mathrm{Si}$ substrate. In the Raman spectrum of graphene, the high-energy double degenerated optical $E_{2 \mathrm{~g}}$ phonon mode is responsible for $\mathrm{G}$ peak whereas the low energy $E_{2 \mathrm{~g}}$ phonon mode is associated with the peak of the bulk graphite located at $42 \mathrm{~cm}^{-1}$ [51]. This feature is caused by a doubly degenerate rigid layer shear mode, involving the relative motion of atoms in adjacent planes [49,52]. Moreover, in twisted FLG (T-FLG), depending on the angle between the layers, the number of phonon modes increases dramatically, leading to significant modification of the phonon spectrum and to the appearance of low energy ZA phonon modes with frequencies of the order of a few $\mathrm{THz}$ [53]. Finally, the presence of a strong electron-phonon coupling in graphene has been invoked to explain the appearance of the superconductivity around $1 \mathrm{~K}$ in twisted bilayer graphene for the magic angle $\theta \approx 1.05^{\circ}[54,55]$. 
While the propagation of phonons from graphene to a massive superconductor is difficult to be detected, in ultrathin superconducting films with thicknesses of the order of the coherence length, the role of phonons generated by graphene could be relevant. In this case, the low-frequency phonon modes of graphene can modify the phonon spectrum of the superconductor and affect its superconducting properties. The main goal of this work is to study the role of graphene layers deposited on $\mathrm{Nb}$ and $\mathrm{NbN}$ superconducting ultrathin films. Both these materials are conventional $s$-wave superconductors well described by the McMillan-Allen-Dynes approximation. The graphene used in this work, is a T-FLG, whose properties were deeply characterized by optical transmittance measurements and by Raman and Xray photoemission spectroscopy (XPS). The thickness of the superconducting films was varied from few up to tens of nanometers and the influence of T-FLG on $T_{\mathrm{c}}$ was studied by measuring the electric transport properties by a resistive method. For the thinnest $\mathrm{Nb}$ layer, the enhancement of $T_{\mathrm{c}}$ reaches the $8 \%$ compared to a single $\mathrm{Nb}$ film. On the contrary, in the case of $\mathrm{NbN}$-based hybrids a clear influence of the graphene layers on $T_{\mathrm{c}}$ was not found. All the obtained results are coherent when discussed in terms of phonon generation in the superconductor due to the presence of graphene.

\section{Experimental}

\section{1. $\mathrm{Nb}$ and $\mathrm{NbN}$ Deposition}

$\mathrm{Nb}$ and $\mathrm{NbN}$ ultrathin superconducting films were deposited on Si substrates by dc UHV magnetron sputtering operating in a base pressure of $1 \times 10^{-8}$ Torr. $\mathrm{Nb}$ films were grown in an Ar pressure of $2.6 \times 10^{-3}$ Torr with a typical deposition rate of $0.33 \mathrm{~nm} / \mathrm{s}$. Since the influence of the graphene layer is expected to be more pronounced for film thicknesses comparable with the superconducting coherence length $\left(\xi_{\mathrm{S}, \mathrm{Nb}} \approx 6 \mathrm{~nm}[56]\right)$, a set of three samples, with thicknesses $d_{\mathrm{Nb}}=10,15$ and $30 \mathrm{~nm}$ was grown. $\mathrm{NbN}$ films were reactively deposited in a mixture of $\mathrm{Ar}$ and $\mathrm{N}_{2}$ with flow rates of 60 and $6.5 \mathrm{sccm}$, respectively. The resulting mixture pressure during the deposition was $3.2 \times 10^{-3}$ Torr with a deposition rate of $0.41 \mathrm{~nm} / \mathrm{s}$. A set of 8 samples was fabricated with thickness in the range $d_{\mathrm{NbN}}=5-65 \mathrm{~nm}$. For the same reason described above, being $\xi_{\mathrm{S}, \mathrm{NbN}} \approx 3 \mathrm{~nm}$ [57], only the films with 
$d_{\mathrm{NbN}} \leq 25 \mathrm{~nm}$ were used to investigate the superconducting properties of the $\mathrm{NbN} /$ graphene hybrids. Thicker films were used for the characterizations reported in the Appendixes. Because all the films of each material were fabricated in the same deposition run, the difference in the superconducting properties is only related to their thickness. Further details about the deposition procedures can be found elsewhere [56,57].

\subsection{Graphene Growth}

Graphene was grown by atmospheric pressure chemical vapor deposition (APCVD) on $\mathrm{Cu}$ substrate with a methane $\left(\mathrm{CH}_{4}\right)$ as a precursor at $1050^{\circ} \mathrm{C}$. In some cases, n-decane $\left(\mathrm{C}_{10} \mathrm{H}_{22}\right)$ was used as a precursor [58], allowing to fabricate a thicker graphene, with a larger number of layers. Samples were fabricated in a mixture of $\mathrm{H}_{2}$ and $\mathrm{N}_{2}$ as a carrier gas with flow rates of 6 and $100 \mathrm{sccm}$, respectively. After the deposition, graphene was transferred on top of the superconducting film employing a wet chemical room temperature transfer process without using a polymeric support [58]. The $\mathrm{Cu}$ support was completely dissolved and the graphene film was gently washed several times in a bath with distilled water prior to the transfer onto the superconductor. Detailed descriptions of the graphene synthesis, transfer approach and graphene characterization can be found elsewhere [58,59].

The resulting samples were labeled with the chemical symbol ( $\mathrm{Nb}$ or $\mathrm{NbN})$ of the superconductor followed by a number indicating its thickness in nanometers. If the film is coated with graphene, the suffix $G$ along with the letter denoting the precursor used during the synthesis of the graphene film (" $\mathrm{m}$ " for methane and " $\mathrm{d}$ " for n-decane) is added. The graphene used for the Nb-based hybrids was always obtained using methane as a precursor. In this case, when labelling the samples, the letter ' $\mathrm{m}$ ' after the value of the thickness is not present. For example, Nb10G is the Superconductor/Graphene (S/G) hybrid made of a 10 -nm-thick niobium film coated with graphene synthesized applying methane, while NbN15Gd and $\mathrm{NbN} 15 \mathrm{Gm}$ stand for $\mathrm{NbN}$ films with a thickness of $15 \mathrm{~nm}$ coated with graphene synthesized using n-decane or methane as a precursor, respectively.

The properties of graphene were characterized by Raman spectroscopy and optical transmittance measurements. The number of graphene layers, $N_{\mathrm{G}}$, was reliably 
obtained from these analyses. It turns out that the graphene which cover $\mathrm{Nb}$ consist of 1-2 numbers of layers. On the contrary, the graphene covering $\mathrm{NbN}$ films has $N_{\mathrm{G}}=3-4$ (7-8) when methane (n-decane) is used as a precursor. These results are shown in Appendix A (Raman data) and Appendix B (Optical transmittance).

The properties of the $\mathrm{Nb} / \mathrm{G}$ and $\mathrm{NbN} / \mathrm{G}$ interfaces were investigated by XPS. The main result of these studies is that the obtained $S / G$ hybrids are complex layered systems having a superconductor/oxide/graphene structure. This means that there is not a direct metallic contact between the superconducting film and graphene and, therefore, superconducting proximity effect can be disregarded in the further considerations. The results of the exhaustive XPS investigation are shown in Appendix C.

Superconducting electric transport properties were measured in a ${ }^{4} \mathrm{He}$ cryostat using a standard $d c$ four-probe technique on unpatterned samples with the electrical contacts put in-line. The samples were biased by a $10 \mu \mathrm{A} d c$ current. When applied, the external magnetic field was perpendicular to the plane of the film and to the direction of the flowing current. The superconducting transition temperature of the samples was estimated at the midpoint of the transition, namely for $R=0.5 R_{10}$, where $R_{10}$ is the resistance at $T=10 \mathrm{~K}$. Temperature in the range from $4 \mathrm{~K}$ to $300 \mathrm{~K}$ was set with an accuracy better than $50 \mathrm{mK}$ and it was measured with a Cernox ${ }^{\mathrm{TM}} \mathrm{CX}-1030$ thermometer located on the holder near the sample. A Lakeshore 340 controller allowed stabilizing temperature with the accuracy of $\pm 5 \mathrm{mK}$ during the measurements.

\section{Superconducting Properties.}

The resistive transition curves measured for samples $\mathrm{Nb} 10$ and $\mathrm{Nb30}$ (open symbols), Nb10G and Nb30G (closed symbols) are shown in Fig. 1(a). A clear enhancement of $T_{\mathrm{c}}$ is evident for both the $\mathrm{Nb} x \mathrm{G}$ hybrids (here $x$ is the $\mathrm{Nb}$ thickness)). By defining $\Delta T_{\mathrm{c}}$ as the difference in $T_{\mathrm{c}}$ of the bare and covered sample, it results that the largest $T_{\mathrm{c}}$ increase is observed for the Nb10G sample, for which it is $\Delta T_{\mathrm{c}}=0.6 \mathrm{~K}$. For the samples Nb15G (not shown here) and Nb30G it is $\Delta T_{\mathrm{c}}=0.1 \mathrm{~K}$. These results are summarized in Fig. 1(b), where the dependence of $T_{\mathrm{c}}$ on $d_{\mathrm{Nb}}$ for the bare films and the $\mathrm{Nb} x \mathrm{G}$ hybrids is compared. The curve relative 
to the $\mathrm{Nb} x \mathrm{G}$ hybrids systematically lies above the one of the single $\mathrm{Nb}$ films, indicating a well-established $T_{\mathrm{c}}$ enhancement, which is larger at low $d_{\mathrm{Nb}}$.
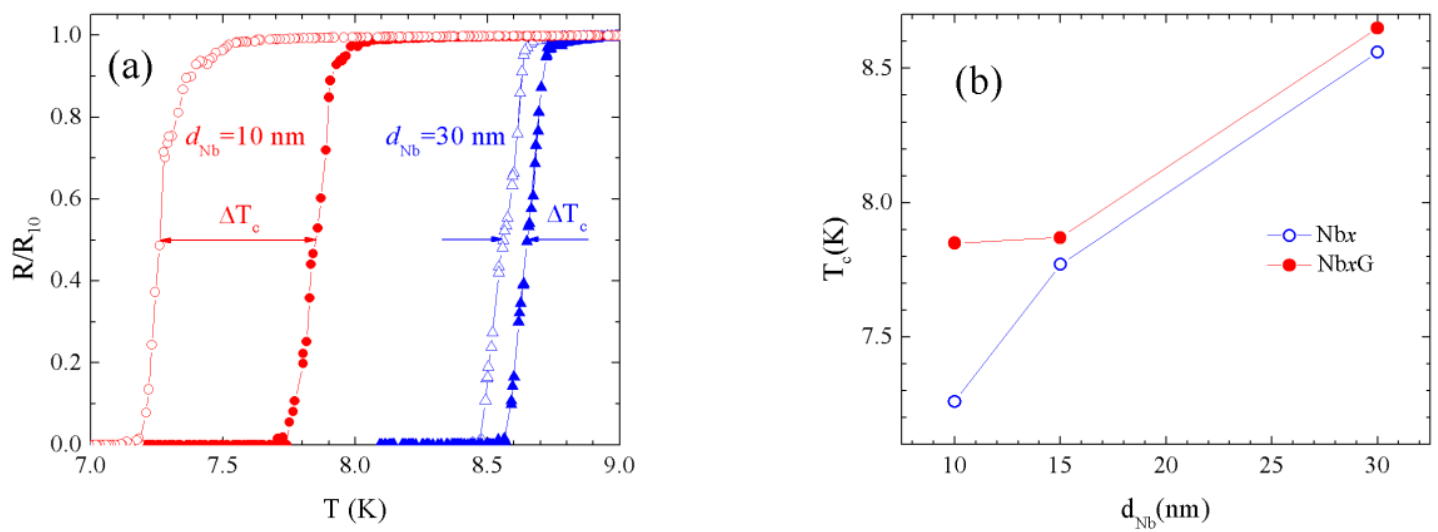

Figure 1. (a) Normalized resistance versus temperature for samples $N b 10$ and Nb10G (red), Nb30 and Nb30G (blue) samples. Open symbols are for bare $\mathrm{Nb}$ films, closed symbols are for NbxG hybrids.(b) Thickness dependence of $T_{c}$ for $\mathrm{Nb}$ films both coated and uncoated by graphene. The letter $x$ stands for the variable value of $d_{N b}$ in $\mathrm{nm}$.

For these samples the temperature dependence of the perpendicular upper critical magnetic field, $H_{\mathrm{c} 2}(T)$, was derived by measuring resistance versus magnetic field, $R(H)$, curves at different temperatures. The results of these measurements for the samples $\mathrm{Nb} 10$ and Nb10G are shown in Figs. 2(a) and 2(b), respectively. $H_{\mathrm{c} 2}$ was determined at the midpoint of the resistive transition. The resulting $H_{\mathrm{c} 2}(T)$ dependences are shown in Fig. 2(c). From these curves the value of the superconducting coherence length, $\xi_{\mathrm{s}}$, can be estimated through the relation $\xi_{\mathrm{S}}=$ $\frac{2^{\frac{1}{2}}}{\pi^{\frac{3}{2}}} \cdot\left[-\frac{\Phi_{0}}{T_{c} \cdot\left(d H_{c 2} / d T\right)_{T=T_{c}}}\right]^{\frac{1}{2}} \quad\left(\Phi_{0}\right.$ being the flux quantum). For Nb10 it is $\mathrm{d} H_{\mathrm{c} 2} / \mathrm{d} T=-0.730 \mathrm{~T} / \mathrm{K}$ and $T_{\mathrm{c}}=7.26 \mathrm{~K}$, which leads to $\xi_{\mathrm{S}}=5.0 \mathrm{~nm}$, while for Nb10G it is $\mathrm{d} H_{\mathrm{c} 2} / \mathrm{d} T=-0.605 \mathrm{~T} / \mathrm{K}$ and $T_{\mathrm{c}}=7.86 \mathrm{~K}$, which gives $\xi_{s}=5.3 \mathrm{~nm}$. The values obtained for $\xi_{\mathrm{S}, \mathrm{Nb}}$ confirm the high quality of ultrathin $\mathrm{Nb}$ films $[56,60]$. 

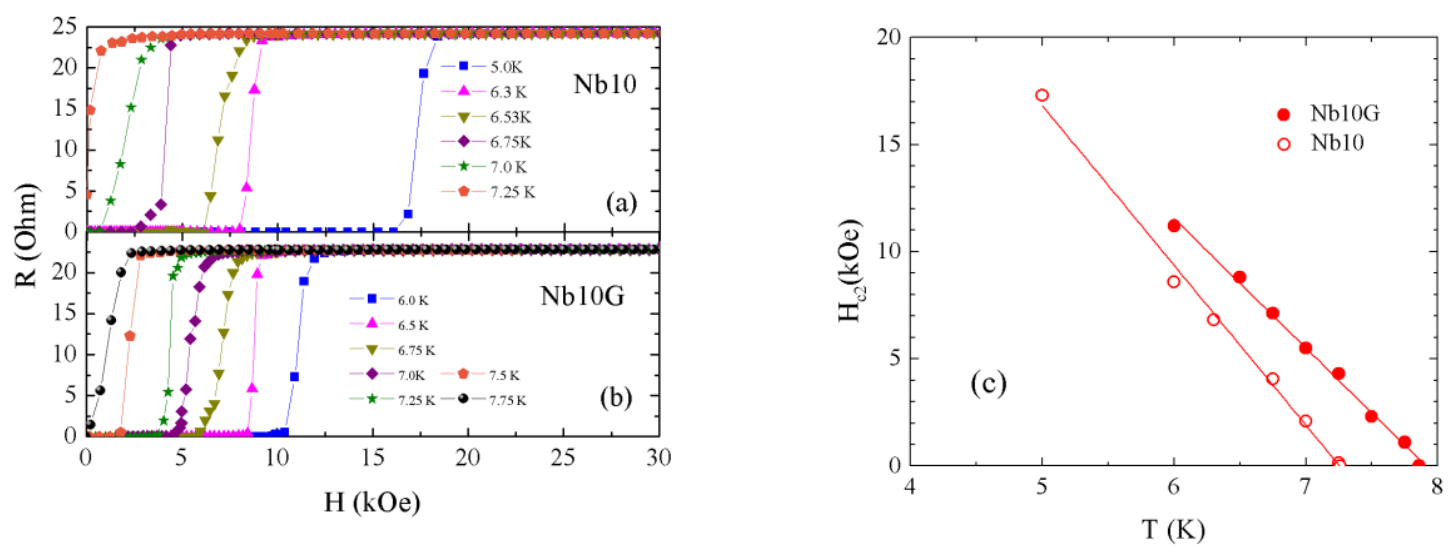

Figure 2. Resistance versus perpendicular magnetic field at different temperatures for samples $\mathrm{Nb10}$ (a) and Nb10G (b). (c) Perpendicular upper critical magnetic field, $H_{c 2}$, versus temperature for $\mathrm{Nb10}$ (open symbols) and Nb10G (closed symbols) samples. Solid lines represent the best linear fit close to $T_{c}$.

A similar study was performed on the NbN/G hybrids. Fig. 3(a) shows the resistive transition curves for a selection of the three $\mathrm{NbN} x \mathrm{G}$-based samples with $d_{\mathrm{NbN}}=7$, 15 and $18 \mathrm{~nm}$. Black symbols indicate the single $\mathrm{NbN} x$ films, while red and blue symbols indicate $\mathrm{NbN} x \mathrm{Gm}$ and $\mathrm{NbN} x \mathrm{Gd}$ hybrids, respectively (here $x$ stands for the $\mathrm{NbN}$ thickness in $\mathrm{nm}$ ).
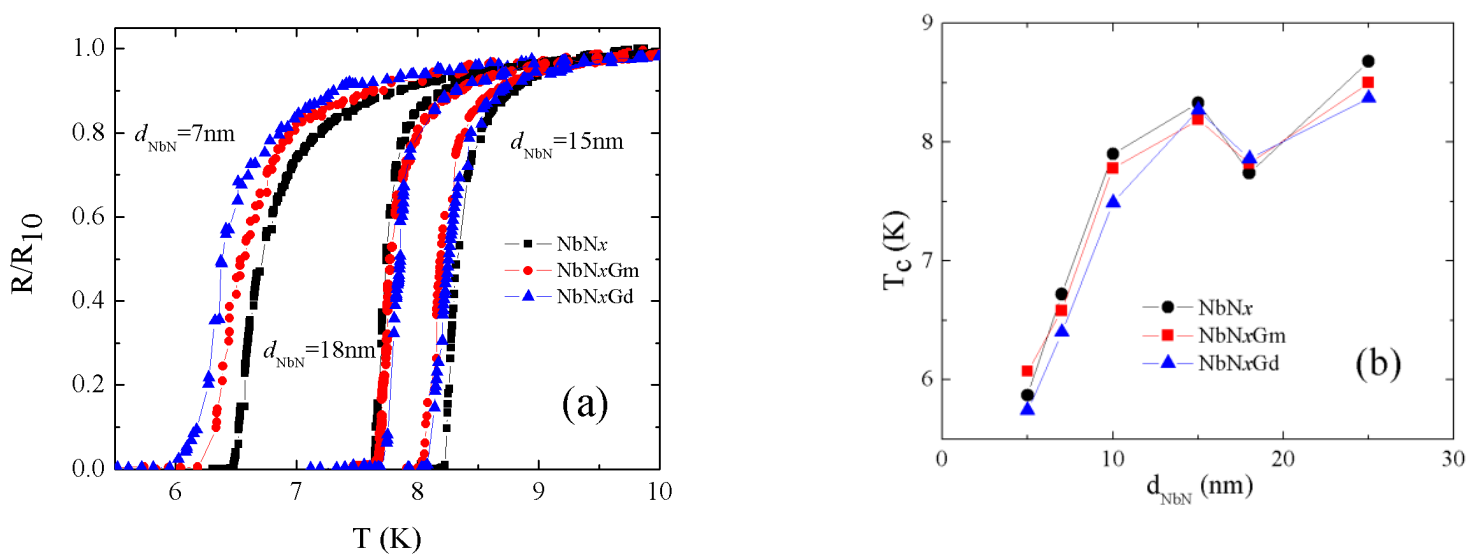

Figure 3. (a) Normalized resistance versus temperature for single NbN films and $N b N / G$ hybrids of different $N b N$ thickness. In the legend $x$ denotes $d_{N b N}$ in nanometers. For details see the text. (b) Thickness dependence of $T_{c}$ for $N b N$ films, both bare and coated by graphene synthesized by using the two different precursors. 
The results obtained for the three set of samples are summarized in Fig. 3(b). Contrary to $\mathrm{Nb}$, the results for $\mathrm{NbN}$ do not to follow a clear trend. The $T_{\mathrm{c}}$ values of the bare films are larger than that of the graphene-coated films for $d_{\mathrm{NbN}}=7,10,15$, and $25 \mathrm{~nm}$. Also, in general the $T_{\mathrm{c}}$ of the films grown with methane as a precursor does not differ much with respect the corresponding $\mathrm{NbN}$ bare samples. In addition, there is a tendency for the samples synthesized with methane to have higher $T_{\mathrm{c}}$ compared to the ones obtained by using n-decane, except for $d_{\mathrm{NbN}}=15$ and $18 \mathrm{~nm}$. For this last thickness, the quality of the films is lower when compared to the other samples of the series, but this does not affect the general discussion.

\section{Discussion}

\section{1. $\mathrm{Nb} / \mathrm{G}$ hybrids}

We consider the effect of graphene on the phonon spectrum as the main reason of the enhancement of $T_{\mathrm{c}}$ observed in ultrathin $\mathrm{Nb}$ films coated with graphene. In the following, we will not give a detailed theoretical analysis but we will mainly underline the physics at play. Let us consider the elementary problem of longitudinal vibrations of a one-dimensional (1D) chain of atoms of mass $M$ connected by springs of rigidity $K$. The mass $M$ is connected to atoms of mass $M_{\mathrm{S}}$ by springs of stiffness $K_{\zeta}$. The $M_{\mathrm{S}}(M)$ atoms are elastically connected to each other by springs of stiffness $K_{\mathrm{S}}(K)$. The chain of $M$ atoms imitates the $\mathrm{Nb}$ film, whereas the two $M_{\mathrm{S}}$ atoms the bilayer graphene. In addition, the weak bond $K_{\zeta}$ between $M$ and $M_{\mathrm{S}}$ atoms also refers to the oxide layer between the superconductor and the graphene. A schematic representation of the 1D chain of the atoms under investigation is shown in Fig. 4(a). 


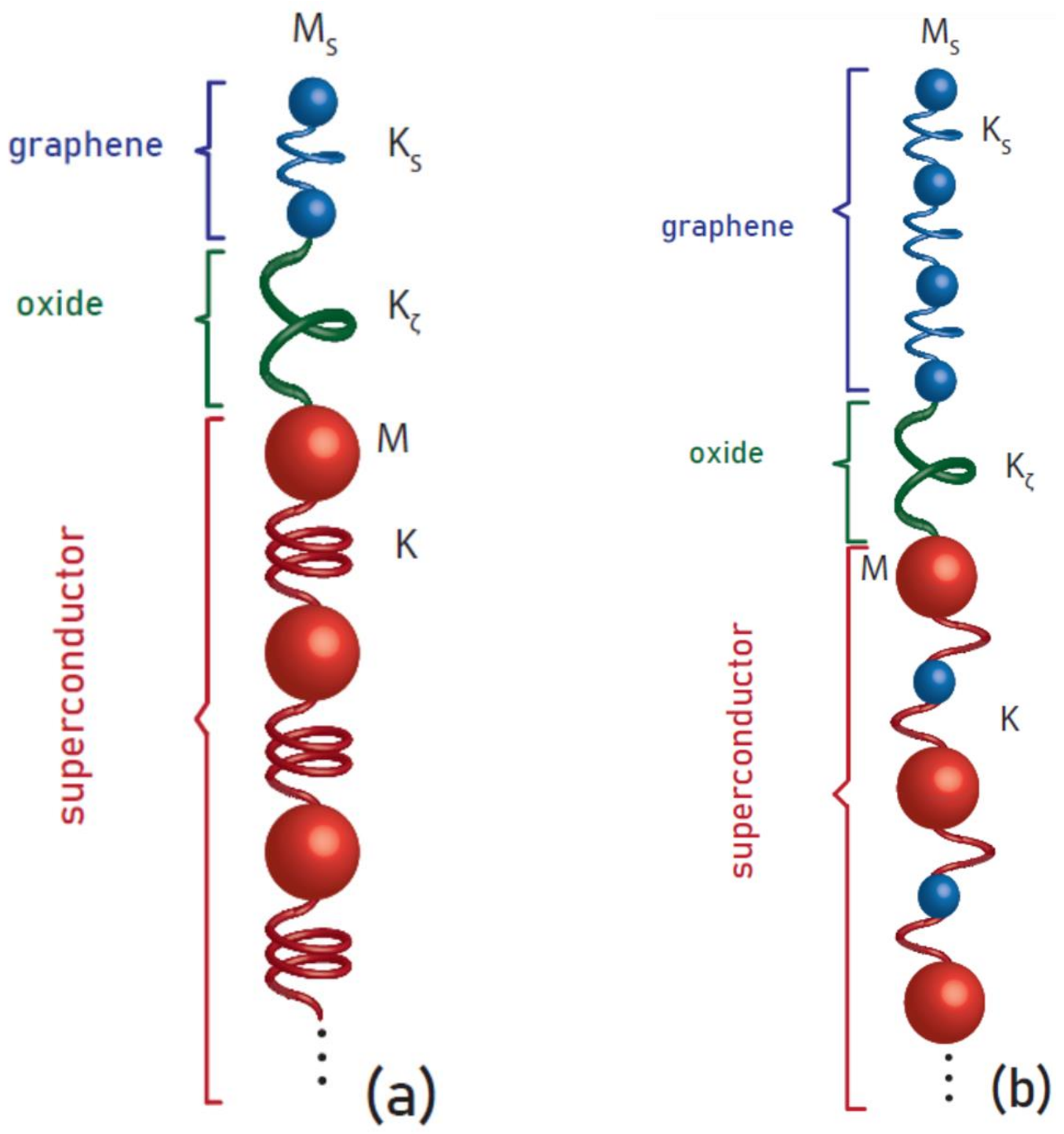

Figure 4. Schematic of the 1D chains by which the systems under investigation are represented. (a) $N b / G$ and (b) NbN/G hybrids. Red circles refer to Nb while blue circles refer to carbon (and nitrogen). For details, see the text.

The solution to this classical problem of an elastic chain of identical atoms is well known, and yields eigenfrequencies $\omega_{n}$ [61]

$$
\omega_{n}=2 \omega_{0}\left|\sin \left(\frac{\pi n}{2 N}\right)\right|,(n=0,1, \ldots, N-1)
$$

where $N$ is the number of atoms in the chain and $\omega_{0}=\sqrt{K / M}$. Next, we scale the parameters of the system under consideration to the parameters of the chain of atoms: $\omega_{s}=\sqrt{K_{s} / M_{s}} \equiv \omega_{0} v_{s}, K_{\zeta} \equiv K \zeta$ and $M_{\mathrm{s}} \equiv M \beta$. Time is measured in unit of 
reciprocal frequencies, $\omega_{0}$. Then, the eigenfrequencies $\omega_{n}$ of the oscillations of the $1 \mathrm{D}$-chain depend on the three parameters $\zeta, \beta$ and $v_{\mathrm{s}}$. The quantity $\beta$ denotes the ratio of masses in the chain, $M_{\mathrm{s}} / M$. We consider it as a fixed value, of the order of the ratio of the atomic masses of carbon and niobium, $\beta \approx 0.13$. Then during the simulations, we vary only two parameters of the system, $\zeta$, which reflects the properties of the oxide layer, and $v_{\mathrm{s}}$, which is referred to the eigenfrequencies of the carbon atoms. We are interested only in the low frequency part of the spectrum, $v_{\mathrm{s}} \ll 1$, which could be associated with a low frequency ZA phonons that, in turn, stimulates superconductivity (see below).

Frequency spectra of a 1D-chain for $v_{\mathrm{s}}=0.05$ and different values of the coupling constant $\zeta$ are shown in the Fig. 5(a) where we plot the eigenfrequencies versus the normalized wave number $\pi n / 2 N$ ( $N$ was set to be equal to 12 , i.e. to 10 atoms with mass $M$ plus 2 atoms with mass $M_{\mathrm{s}}$ ). For comparison, the spectrum of a 1D-chain consisting of 12 atoms with a mass of $M$ each atom is shown as the black solid curve in Fig. 5(a).

In Fig. 5(b), for the sake of convenience, we present the obtained set of frequencies. To better underline the role played by the two additional atoms, we show the set of frequencies calculated for a 1D-chain made of 10 atoms with mass $M$ and those calculated for a 1D-chain made of 12 atoms with mass $M$. It follows from this result [as also seen from Fig. 5(a)] that the edge (surface) perturbations have a small effect on the high frequency part of the spectrum. Then, an increase in the PhDOS for relatively low values of $\omega$ is observed. Rectangles in Fig. 5(b) mark regions of the increased PhDOS. We believe that peaks in the PhDOS in the lowfrequency region will also appear in the real 3D sample. 

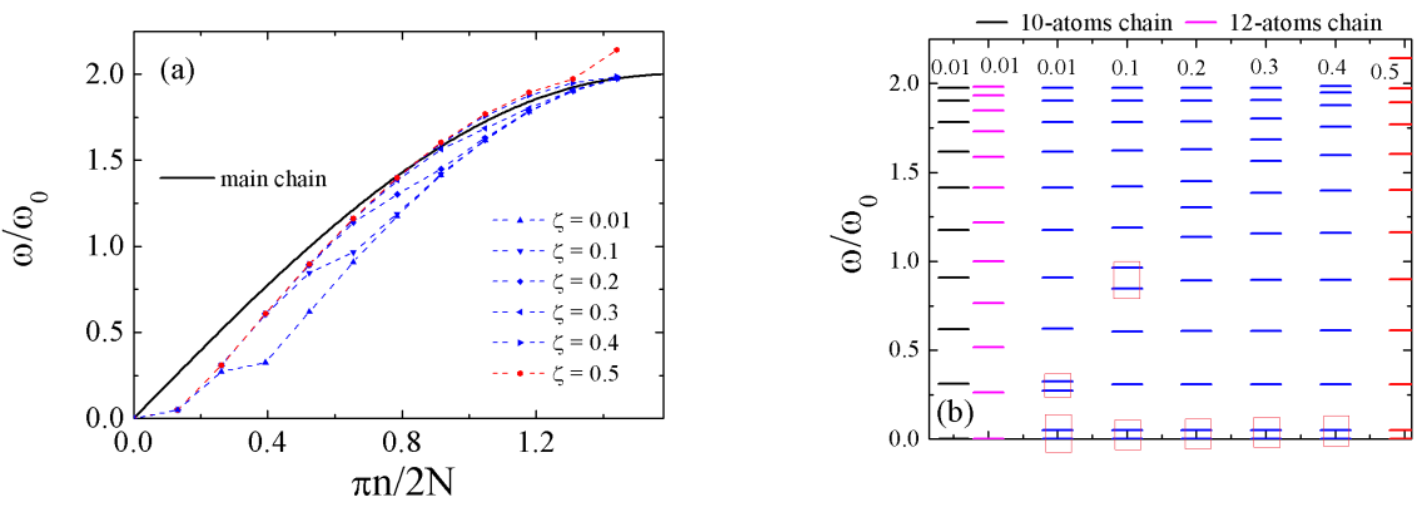

Figure 5. Frequency spectra for a $1 D$-chain of atoms for $v_{s}=0.05$ and different coupling constants $\zeta$. (a) Eigenfrequencies versus the reduced wave number, $\pi n / 2 N$ (see text for details). (b) The obtained set of frequencies. Numbers denote $\zeta$ values. Rectangles mark regions of eigenfrequencies of the chain induced by the $M_{S}$ atoms. Black (magenta) bars are for $N=10$ (12) niobium atoms in the $1 D$ chain.

From this result it follows that, for a small relative frequencies $v_{\mathrm{s}}$ and weak coupling $\zeta$, the PhDOS in the region of small frequencies $\omega$ in the $\mathrm{Nb} / \mathrm{G}$ hybrids could be increased. Considering that for $\mathrm{Nb}$ the electron-phonon coupling parameter $\alpha^{2}(\omega)$ is almost constant in the frequency range $2-7 \mathrm{THz}$ [11], the increase in the PhDOS implies a decrease in the McMillan $\left\langle\omega^{2}\right\rangle$ value, which in turn determines an enhancement of the superconducting critical temperature. This is exactly the classical effect of the softening of the phonon spectrum, which was intensively discussed earlier in the literature [62]. If we reasonably set $2 \omega_{0} \sim T_{D} \sim 300 \mathrm{~K}$, the significant softening of the phonon spectrum occurs at $\omega_{\mathrm{s}}$ of the order of few Terahertz. This is in good agreement with the range of the phonon spectra needed to enhance the superconductivity and this result gives a support in favour of the reasonableness of the above considerations, despite the oversimplified adopted one-dimensional model.

To investigate the influence of the eigenfrequencies of the carbon atoms on the frequency spectra of the entire chain, we look at the frequency spectra for a wide range of $v_{\mathrm{s}}$. This result is presented in Fig. 6 for two values of the coupling constant $\zeta=0.01$ and $\zeta=0.1$. For each value of $\zeta$, $v_{\mathrm{s}}$ was varied in a wide range, i.e. $0.01<v_{\mathrm{s}}<1$. It follows from Fig. 6 that, contrary on what was previously 
obtained when the coupling constant $\zeta$ was changed, the influence of $v_{\mathrm{s}}$ on the frequency spectra is not so crucial. The main deviations occur in the lower frequency region of the spectra, which again could favor the enhancement of $T_{\mathrm{c}}$.
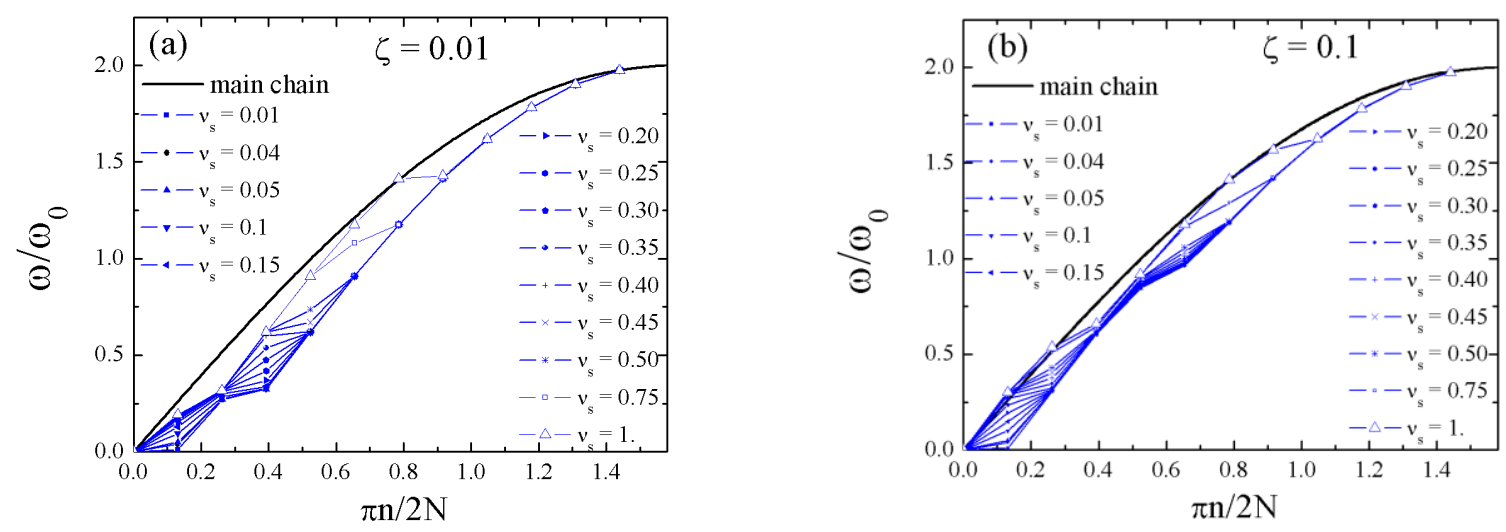

Figure 6. Frequency spectra for a $1 D$-chain of 10 atoms of mass $M$ terminated with 2 atoms of mass $M_{S}$ for two different coupling constants $\zeta$ : (a) $\zeta=0.01 ;(b)$ $\zeta=0.1$. For each value of $\zeta$ results for a wide range of $v_{s}\left(0.01<v_{s}<1\right)$ are shown.

\section{2. $\mathrm{NbN} / \mathrm{G}$ hybrids}

Now we move to the analysis of the NbN/G hybrids for which, as observed experimentally, the values of $T_{\mathrm{c}}$ are less affected by the presence of graphene. We model the system as a 1D-chain made of $10 \mathrm{Nb}$ atoms attached to 10 Nitrogen atoms. This diatomic chain is terminated with 4 Carbon atoms. A schematic of the structure is shown in Fig. 4(b). It is assumed that the atomic masses of C and N, which are neighbors in the periodic table of elements, are equal.

The frequency spectra of a chain made by 10 pairs of atoms with mass $M$ and $M_{\mathrm{S}}$ atoms terminated by 4 atoms with mass $M_{\mathrm{S}}$ are shown in Fig. 7(a) where both acoustic and optic branches are plotted. Unlike the case of $\mathrm{Nb} / \mathrm{G}$ hybrids, there is a middle range of $\omega$, that is most susceptible to the deformation. In addition, the optical part of the spectrum, which contributes approximately $20 \%$ to the value of the effective coupling constant (thorough calculations were done in Ref. [63]) is not subject to any changes. This insensitivity is a well-known effect for a 
crystalline structure formed by heavy $(\mathrm{Nb})$ and light $(\mathrm{N})$ atoms [61]. The acoustic branch is set by the parameters of the heavy sub-lattice and the optic branch is defined by the parameters of the light sub-lattice $[11,61,63]$. Therefore, it is clear that if a lattice of light atoms is added to the main structure once the optic branch is formed this branch will not change.

In Fig. 7(b) the obtained set of frequencies are shown. By black and magenta bars, we mark the eigenfrequencies of a main chain consisting only of pairs of atoms with masses $M$ and $M_{\mathrm{S}}$. Black (magenta) bars refer to the case $N=10$ (14). It is clear that while the surface perturbations do not influence the high frequency region of the spectrum, deviations occur in the intermediate region. The effect is more pronounced for smaller $\zeta$ (i.e. for a greater influence of the oxide layers). However, even though in a slightly different frequency range, the effect of softening of the phonon spectra is observed.

The influence of the terminated atoms having mass $M_{\mathrm{S}}$ on the eigenfrequencies of the spectrum of the entire chain have been investigated and the results are shown in Fig. 8 for two values of the oxide coupling strength $(\zeta=0.01$ and $\zeta=0.1)$. For each value of $\zeta$, which describes the properties of the oxide layer, the optic and acoustic branches are calculated for a wide range of values of $v_{\mathrm{s}}$. As in the case of the $\mathrm{Nb} / \mathrm{G}$ hybrids, the effect of the carbon is more pronounced for smaller values of $v_{\mathrm{s}}$, while greater deviations occur in the intermediate frequency range.
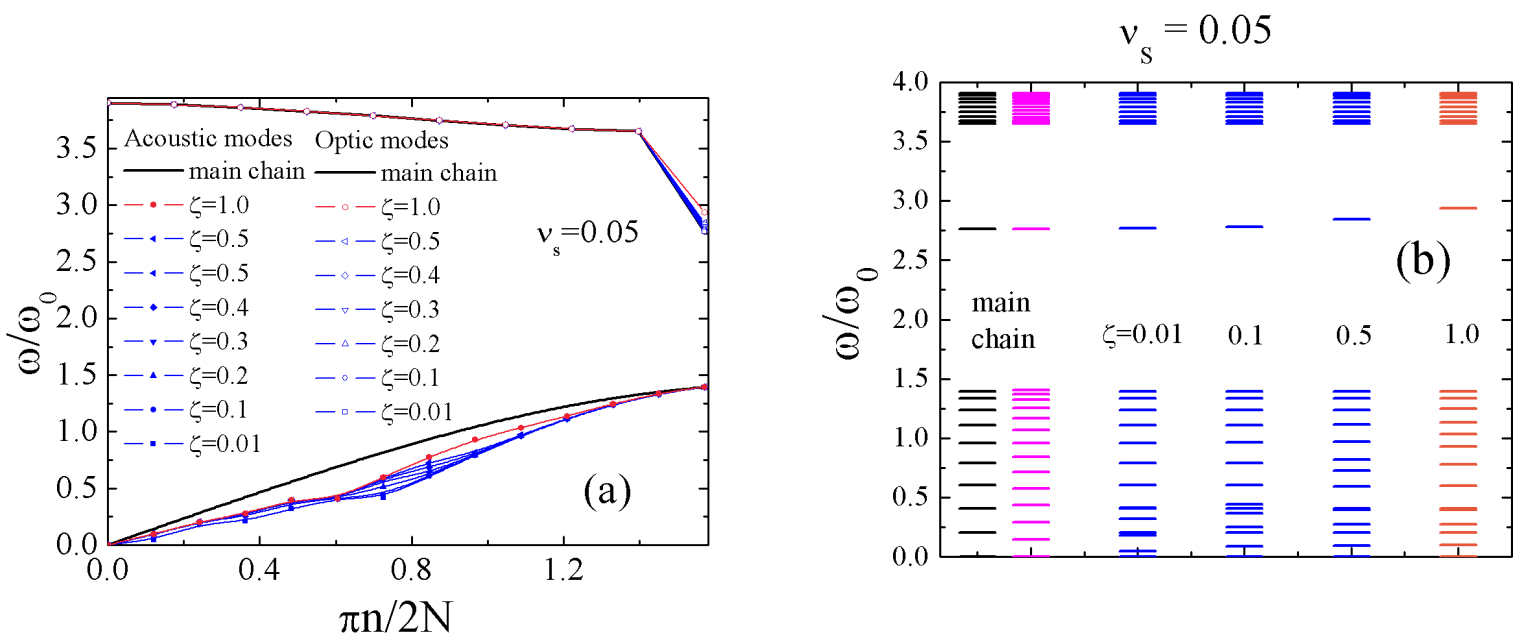

Figure 7. Frequency spectra for a 1D-diatomic chain of atoms calculated for $v_{s}=0.05$ and different values of the coupling constant $\zeta$. Both acoustic and optic 
branches are shown. Panel (a): Eigenfrequencies versus the reduced wave number. Panel (b): The obtained set of frequencies. Black (magenta) bars are for $N=10(14)$ pairs of atoms with masses $M$ and $M_{S}$ in the $1 D$-chain.

However, this theoretical prediction does not explain the observation that, in the case of $\mathrm{NbN} / \mathrm{G}$ hybrids, the presence of the graphene does not cause an increase of the superconducting critical temperature with respect to bare $\mathrm{NbN}$ films. This, we believe, may be due to the peculiar $\alpha^{2}(\omega)$ dependence in $\mathrm{NbN}$. Indeed, in the case of $\mathrm{Nb}$ the quantity $\alpha^{2}(\omega)$ is almost frequency independent [11]. Therefore, if the spectral Eliashberg function $\alpha^{2}(\omega) F(\omega)$ is known, the PhDOS can be evaluated easily. In particular, the increase in the PhDOS leads unambiguously to the increase of the effective electron-phonon coupling constant $\lambda$ [Eq. (2)]. This, in turn, causes an increase of $T_{\mathrm{c}}$.
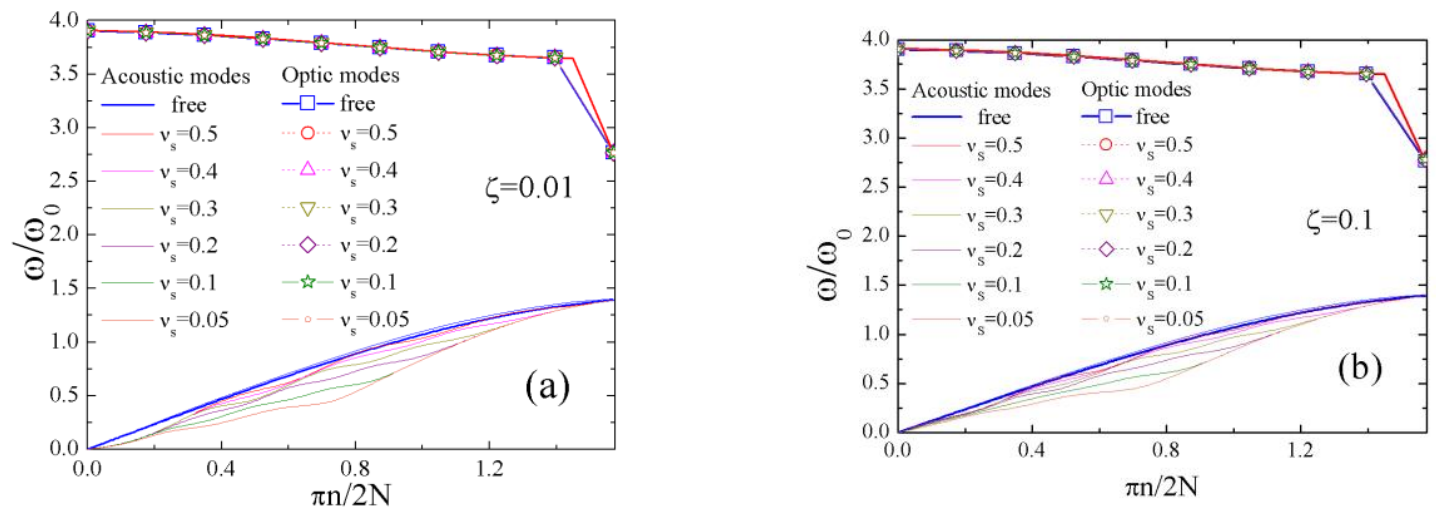

Figure 8. Frequency spectra for a $1 D$ chain of atoms with masses $M$ and $M_{S}$ terminated with 4 atoms with mass $M_{s}$ for two different values of the coupling constant $\zeta .:(a) \zeta=0.01$ and $(b) \zeta=0.1$. For each coupling constant spectra for $a$ wide range of $v_{s}\left(0.05<v_{s}<1\right)$ have been studied. In the figures, the optic and acoustic modes are plotted.

In the case of $\mathrm{NbN}$, it is not possible to separate the role played on $T_{\mathrm{c}}$ by the phonon spectrum, $F(\omega)$, and by electron-phonon interaction spectrum, $\alpha^{2}(\omega)$ [10]. In fact, for this material, $\alpha^{2}(\omega)$ is a more strongly oscillating function with respect to $F(\omega)$, in particular in the frequency range $2-7.5 \mathrm{THz}$ of the acoustic branch. Moreover, the function $\alpha^{2}(\omega)$ oscillates with large amplitude [63]. Therefore, the effect of variations in the function $F(\omega)$ caused by an external source is largely 
hidden due to these oscillations. In this regards we note that the higher critical temperature of the $\mathrm{NbN}$ is due to a higher density of electronic states rather than a PhDOS (the electronic DOS of $\mathrm{NbN}$ exceeds the DOS for bare $\mathrm{Nb}$ because of the contribution of the $\mathrm{N}-2 \mathrm{p}$ states in addition to the $\mathrm{Nb}-4 \mathrm{~d}$ states) $[63,64]$. Therefore, due to the oscillating nature of the quantity $\alpha^{2}(\omega) F(\omega) / \omega$, its integral, which defines $\lambda$, does not significantly affect the behavior of $T_{\mathrm{c}}$. In fact, since the modification of the phonon spectrum occurs in a wide frequency range (see Figs. 7 and 8), a positive contribution to $\lambda$ in a certain band of the $F(\omega)$ spectrum may be compensated only by a negative contribution coming from the remaining part of the spectrum. As a result, the superconducting state of $\mathrm{NbN}$ films is not significantly affected by phonon pumping from graphene, even when the number of graphene layers is increased.

\section{Conclusions}

In conclusion, the superconducting properties of Superconductor/Graphene hybrids based on $\mathrm{Nb}$ and $\mathrm{NbN}$ ultrathin films have been studied. Raman spectroscopy measurements showed that the number of graphene layers in the systems were 1-2 for $\mathrm{Nb} / \mathrm{G}$ and $2-4$ or $7-8$ for $\mathrm{NbN} / \mathrm{G}$, depending, in this last case, on the deposition parameters and the precursor used during the graphene synthesis. XPS analysis revealed the presence of oxide (oxide/oxynitride) layers between $\mathrm{Nb}(\mathrm{NbN})$ and graphene thus excluding the presence of metallic proximity coupling. In the case of $\mathrm{Nb} / \mathrm{G}$ hybrids the value of $T_{\mathrm{c}}$ is larger with respect to bare $\mathrm{Nb}$ films. For $d_{\mathrm{Nb}}=10 \mathrm{~nm}$, the increase was of the order of the $8 \%$. On the contrary, for NbN/G hybrids, the values of $T_{\mathrm{c}}$ are not influenced by the presence of graphene. The obtained experimental results have been explained by a qualitative analysis of the effect of the graphene phonon spectrum on the Eliashberg spectral function of a superconductor. This effect has been clarified by calculations of the elementary model of the 1D-chain of atoms of a superconductor terminated by carbon atoms. The calculated eigenfrequencies of the chain definitely revealed a modification of the spectrum in the low-frequency region. In the case of the $\mathrm{Nb} / \mathrm{G}$ system, this result leads to the enhancement of $T_{\mathrm{c}}$ because of the increase of the PhDOS at low frequencies (the phonon spectrum softening). On the contrary, due to the strong oscillating character of the quantity $\alpha^{2}(\omega)$ in $\mathrm{NbN}$, the appearance of new 
eigenfrequencies in the spectrum of $\mathrm{NbN} / \mathrm{G}$ hybrids is not enough to produce an enhancement of the critical temperature in this system.

\section{Acknowledgements}

SLP and IVK acknowledge the financial support of the "Improving the Competitiveness Program" of the National Research Nuclear University (MEPhI).

\section{Appendix A. Raman Spectroscopy}

Raman spectroscopy was performed using the Confotec NR 500 confocal microRaman spectrometer with a $473 \mathrm{~nm}$ excitation wavelength. The resolution of the spectrometer was $3 \mathrm{~cm}^{-1}$.

In Fig. A.1(a) we show the Raman spectrum of the sample NbN25Gm. The observation of the characteristic $\mathrm{G}$ and $2 \mathrm{D}$ bands, their positions $\left(1590 \mathrm{~cm}^{-1}\right.$ and $2672 \mathrm{~cm}^{-1}$, respectively), shape and intensity ratio $\left(I_{2 \mathrm{D}} / I_{\mathrm{G}}=1.03\right)$ revealed the formation of a few layer graphene [58].
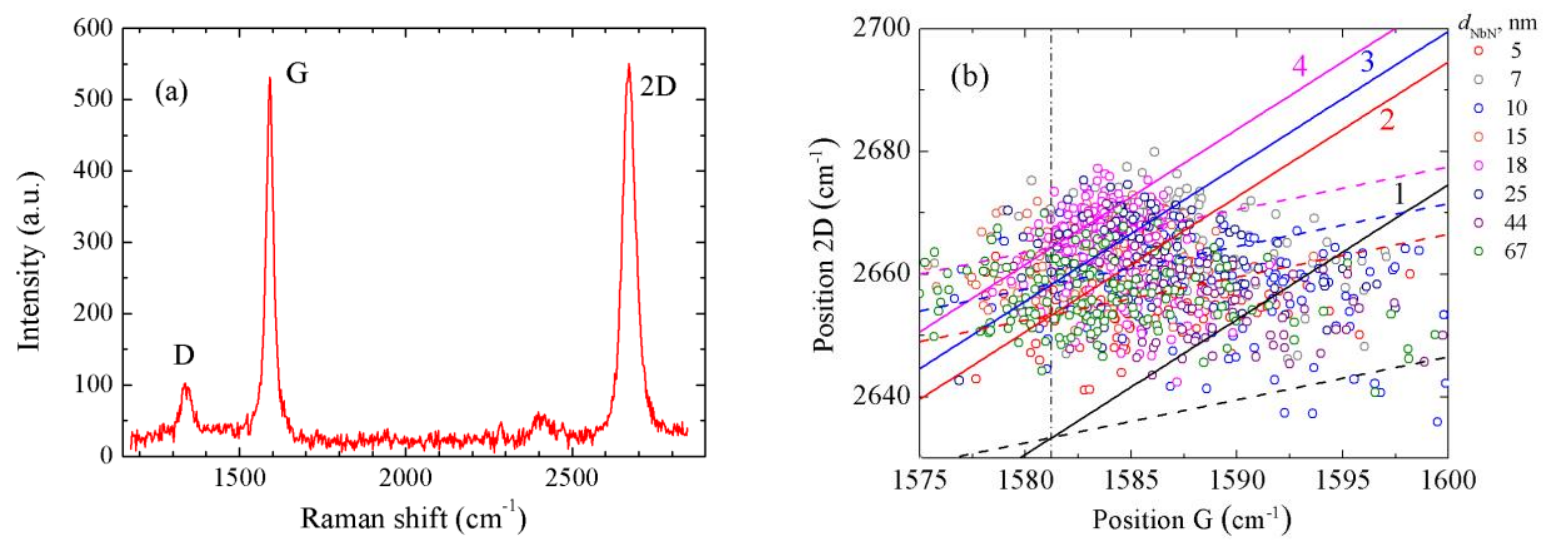

Figure A.1. (a) Raman spectrum of the sample NbN25Gm. (b) Experimental dependencies of the position of the 2D-peak versus the position of the G-peak for the samples of the NbNxGm series (open circles). Solid (dashed) lines with the slope 0.2 (0.7) correspond to a constant carrier (strain) concentration in the graphene. Numbers close to solid lines indicate the number of layers in the FLG while solid and dotted lines with the same color refer to the same number of layers in the FLG. Intersections of the vertical dashed-dotted line with the solid lines are the points for an ideal graphene. Their positions are adapted from Ref. 65. 
By acquiring the Raman spectrum at each point of the sample, we constructed the Raman spectra maps, which gives additional information about the properties and homogeneity of graphene layers. Subsequently, the obtained Raman spectra maps were processed and the dependencies of the integral intensity of the position of the $2 \mathrm{D}$ peak on the position of the $\mathrm{G}$ peak were constructed. This procedure was performed for all the samples. As an example, in Fig. A.1(b) the results for the samples of the $\mathrm{NbN} x \mathrm{Gm}$ series are shown. This plot gives information about stresses in graphene as well as about the number of graphene layers present in the system [66,67]. For a SLG, the positions of the $2 \mathrm{D}$ and $\mathrm{G}$ peaks are governed both by strain and charge transfer, where strain "moves" data points away from the positions $I_{\mathrm{G}}=1581 \mathrm{~cm}^{-1}$ and $I_{2 \mathrm{D}}=2635 \mathrm{~cm}^{-1}$ (typical of an ideal unstrained and undoped graphene) along the 2.2 slope line. On the contrary, hole doping "moves" it along the line with the 0.7 slope in the "position of the $2 \mathrm{D}$ peak - position of the $\mathrm{G}$ peak" plot $[58,68]$. In the case of a FLG, this tendency in the positions of the $2 \mathrm{D}$ and $\mathrm{G}$ peaks persists. Moreover, due to the multilayered nature of the graphene, the 2D position is gradually blue shifted [69]. Solid (dashed) lines in Fig. A.1(b) correspond to the slope $2.2(0.7)$ in this coordinates. Different colors of lines are for different $N_{\mathrm{G}}$ values. As one may see, although graphene is not perfectly homogeneous, most of the experimental points for the $\mathrm{NbN} x \mathrm{Gm}$ hybrids are associated with $N_{\mathrm{G}}=2-4$. Moreover, the shift of the positions of the $\mathrm{G}$ and $2 \mathrm{D}$ peaks is reasonably caused by an increase of the strain in the graphene layers. The non-uniformity of the number of the graphene layers, observed on macroscopically large area, $20 \times 20 \mu \mathrm{m}^{2}$, could be related to the APCVD technique used for the growth process. On the contrary, $N_{\mathrm{G}}$ is in the range of 6-8 for $\mathrm{NbN} x \mathrm{Gd}$ series. Finally, the results for the $\mathrm{Nb} x \mathrm{G}$ series are similar except for values obtained for $N_{\mathrm{G}}$ which, due to a slightly different set of a technological parameters using during the APCVD process, vary between 1 and 2 .

\section{Appendix B. Optical Transmittance}

The measurements of optical transmittance, $\mathcal{T}$, gives information about the value of $N_{\mathrm{G}}$. $\mathcal{T}$ was measured by a PROSCAN MC-121 spectrometer performing a quantitative analysis on a probed circular area with a diameter of $\approx 0.5 \mathrm{~cm}$. For 
these measurements, graphene samples synthesized under the same conditions used for the graphene of the $S / G$ hybrids were analyzed. But this time graphene was transferred to a glass transparent substrate. In Fig. B.1, we show $\mathscr{T}$ of these samples recorded in the $400-900 \mathrm{~nm}$ range. At the wavelength of $550 \mathrm{~nm}$, the graphene used to cover $\mathrm{Nb}$ films has $\mathcal{T}=96.12 \%$ while the transmittance of the graphene subsequently transferred on $\mathrm{NbN}$ is $92.92 \%$ and $83.46 \%$ when methane and n-decane are used as a precursor, respectively. Based on these numbers $N_{\mathrm{G}}$ can be estimated [65]. It turns out that the graphene used to cover $\mathrm{Nb}$ films has $N_{\mathrm{G}}$ between $1(\mathscr{T}=97.7 \%)$ and $2(\mathscr{T}=95.45 \%)$. On the contrary, the graphene used for the $\mathrm{NbN} / \mathrm{G}$ hybrids has $N_{\mathrm{G}}$ between $3(\mathscr{T}=93.25 \%)$ and $4(\mathscr{T}=91.11 \%)$ when synthesized with methane as a precursor, while $N_{\mathrm{G}}$ varies between $7(\mathscr{T}=84.96 \%)$ and $8(\mathscr{T}=83.01 \%)$ with n-decane as a precursor. The difference observed in $N_{\mathrm{G}}$ in the last two cases may be due to the different parameters used during the synthesis of the graphene.

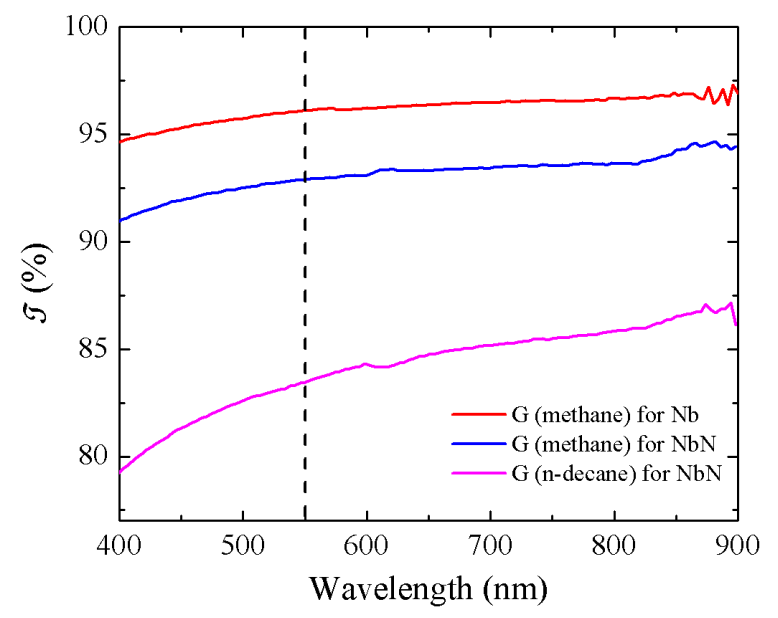

Figure B.1. Optical transmittance $\mathcal{T}$ of different graphene samples transferred on a transparent glass substrate. The red line refers to the sample synthesized under the same conditions used for the graphene subsequently transferred to the $\mathrm{Nb}$ film. Blue (magenta) line refers to the sample grown with a methane (n-decane) as a precursor in the same conditions of the graphene subsequently transferred to the $\mathrm{NbN}$ film. The vertical line indicates the wavelength, $550 \mathrm{~nm}$, at which $\mathfrak{T}$ was estimated. 


\section{Appendix C. X-ray Photoelectron Spectroscopy (XPS)}

A Thermo Scientific ESCALAB 250Xi spectrometer with monochromatic Al Ka radiation $(1486.6 \mathrm{eV})$ was used for XPS measurements. The measurements were performed on as-prepared samples. The base pressure in the analytical chamber was lower than $2 \times 10^{-7} \mathrm{~Pa}$. The $40 \mathrm{eV}$ and $20 \mathrm{eV}$ pass energy values of a hemispherical electron energy analyzer were used for the survey and for the recording of high-resolution spectra, respectively. The energy scale of the system was calibrated with respect to $\mathrm{Au} 4 \mathrm{f}_{7 / 2}, \mathrm{Ag} 3 \mathrm{~d}_{5 / 2}$, and $\mathrm{Cu} 2 \mathrm{p}_{3 / 2}$ peak positions. Further information about the XPS equipment can be found elsewhere [58,70]. XPS measurements were performed on samples $\mathrm{Nb} 10$ and $\mathrm{NbN} 25$, as well as on the corresponding ones coated by graphene, namely samples Nb10G, NbN25Gm and NbN25Gd. In Fig. C.1(a) we show the XPS survey spectrum for the single Nb film, Nb10. The surface composition of the sample reveals the presence of niobium, oxygen and carbon. In addition, a small peak of $\mathrm{SiO}_{2}$ coming from the substrate is present. The niobium presents all the peaks known from literature, i.e. $\mathrm{Nb3s}, \mathrm{Nb3p}, \mathrm{Nb3d}, \mathrm{Nb} 4 \mathrm{~s}$ and $\mathrm{Nb} 4 \mathrm{p}$ [71]. The presence of the small amount of carbon (C1s peak at the bonding energy $E_{\mathrm{b}}=284.48 \mathrm{eV}$ [72]) could be attributed to the usual atmospheric carbon contamination.

The survey spectrum of the sample Nb10G shown in Fig. C.1(b) shows a smaller amplitude for all the $\mathrm{Nb}$ peaks. The $\mathrm{C} 1 \mathrm{~s}$ peak becomes stronger, indicating the presence of graphene. The appearance of a small peak of nitrogen could be attributed to the contamination connected to the wet transfer process. 


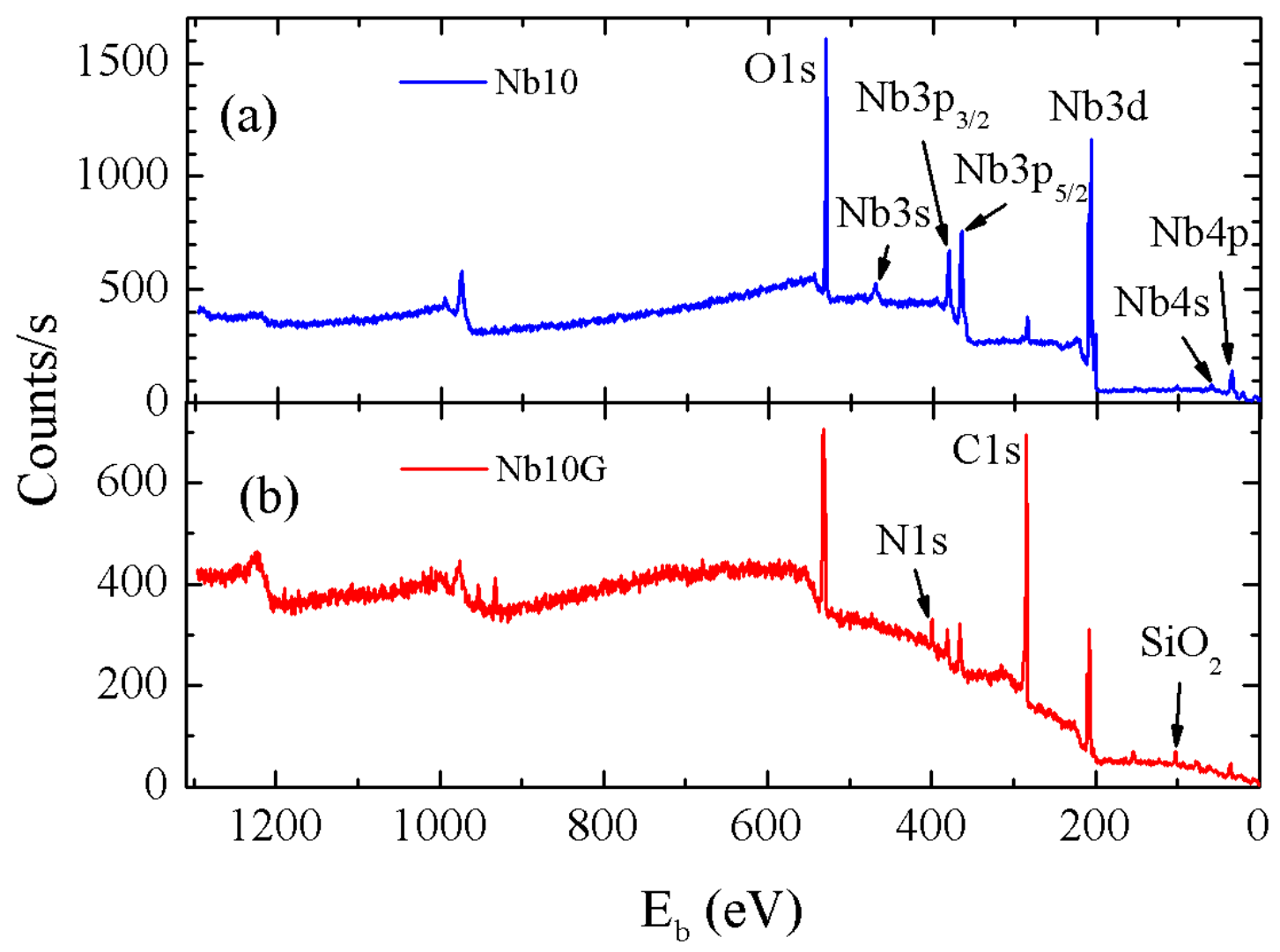

Figure C.1. Survey XPS spectra for the samples $N b 10(a)$ and $N b 10 G(b)$.

Survey spectrum of NbN25 is shown in Fig. C.2(a). The main difference with respect to the case of $\mathrm{Nb10}$ is the presence of a strong nitrogen peak $\left(E_{\mathrm{b}}=397.1 \mathrm{eV}\right.$ [71]), which is naturally expected for a $\mathrm{NbN}$ film. After the graphene transfer, amplitude of the $\mathrm{Nb}$ peaks decrease significantly, as in the case of the sample $\mathrm{Nb} 10 \mathrm{G}$. Here, the carbon peak $\mathrm{C} 1 \mathrm{~s}$ becomes dominant, the niobium peaks are significantly reduced and the nitrogen peak is almost invisible. The stronger suppression of the oxygen peak for NbN25Gd [Fig. C.2(c)] may be reasonably explained by the greater thickness of the graphene in this sample with respect to the case of NbN25Gm [Fig. C.2(b)].

High resolution XPS (HR XPS) C1s core level spectra of the samples Nb10G, $\mathrm{NbN} 25 \mathrm{Gm}$ and $\mathrm{NbN} 25 \mathrm{Gd}$ are shown in Fig. C.3 where the sharp asymmetric peak typical of the graphene is clearly present. The asymmetry of the spectrum is more 
pronounced for the $\mathrm{Nb} 10 \mathrm{G}$ sample due to the lower value of $N_{\mathrm{G}}$ in this sample. The small peak at $E_{\mathrm{b}}=288.2 \mathrm{eV}$ is typical of the $\mathrm{O}-\mathrm{C}=\mathrm{O}$ bonds [73].

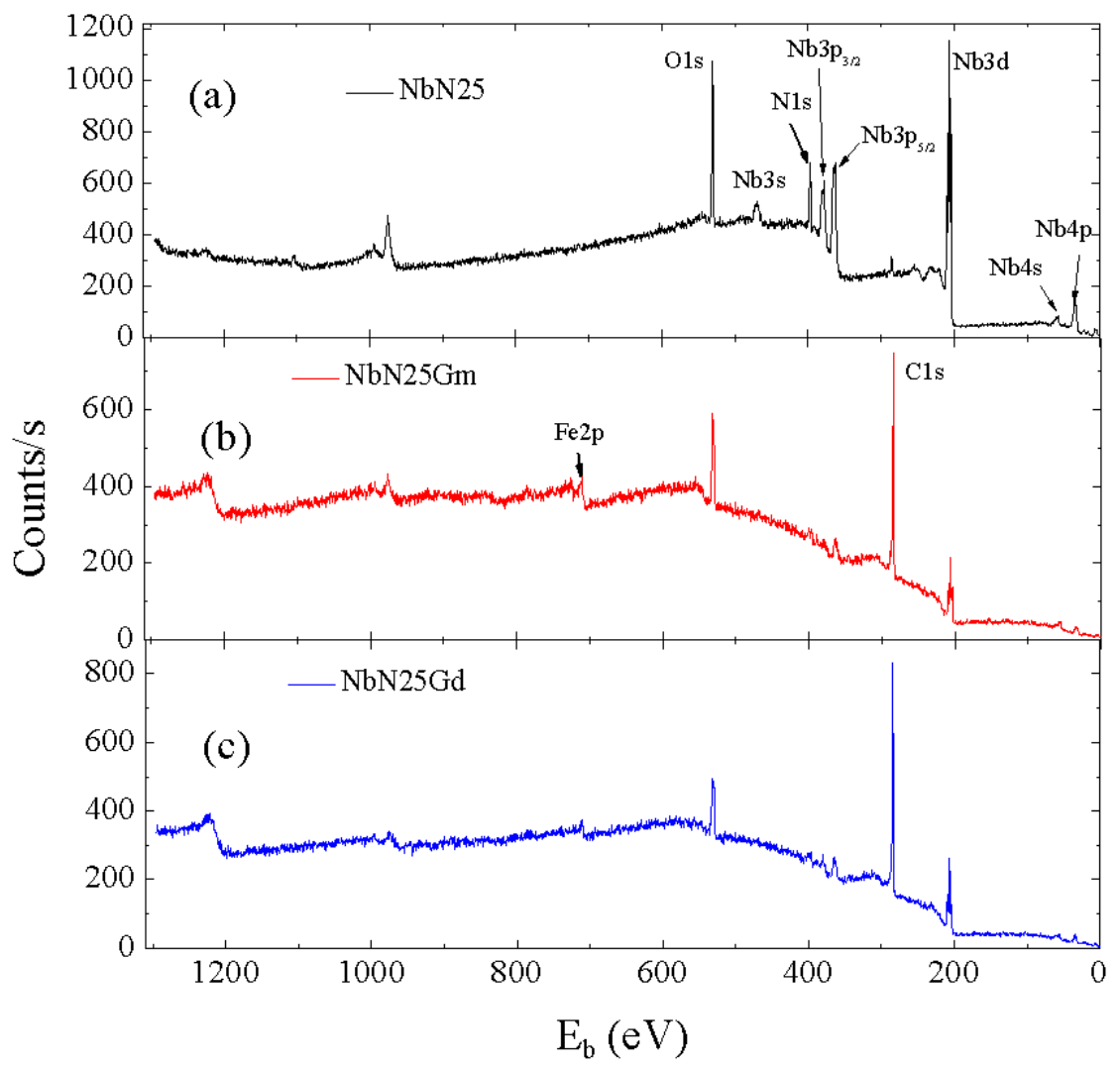

Figure C.2. Survey XPS spectra for the sample NbN25 (a), NbN25Gm (b) and $N b N 25 G d(c)$. 


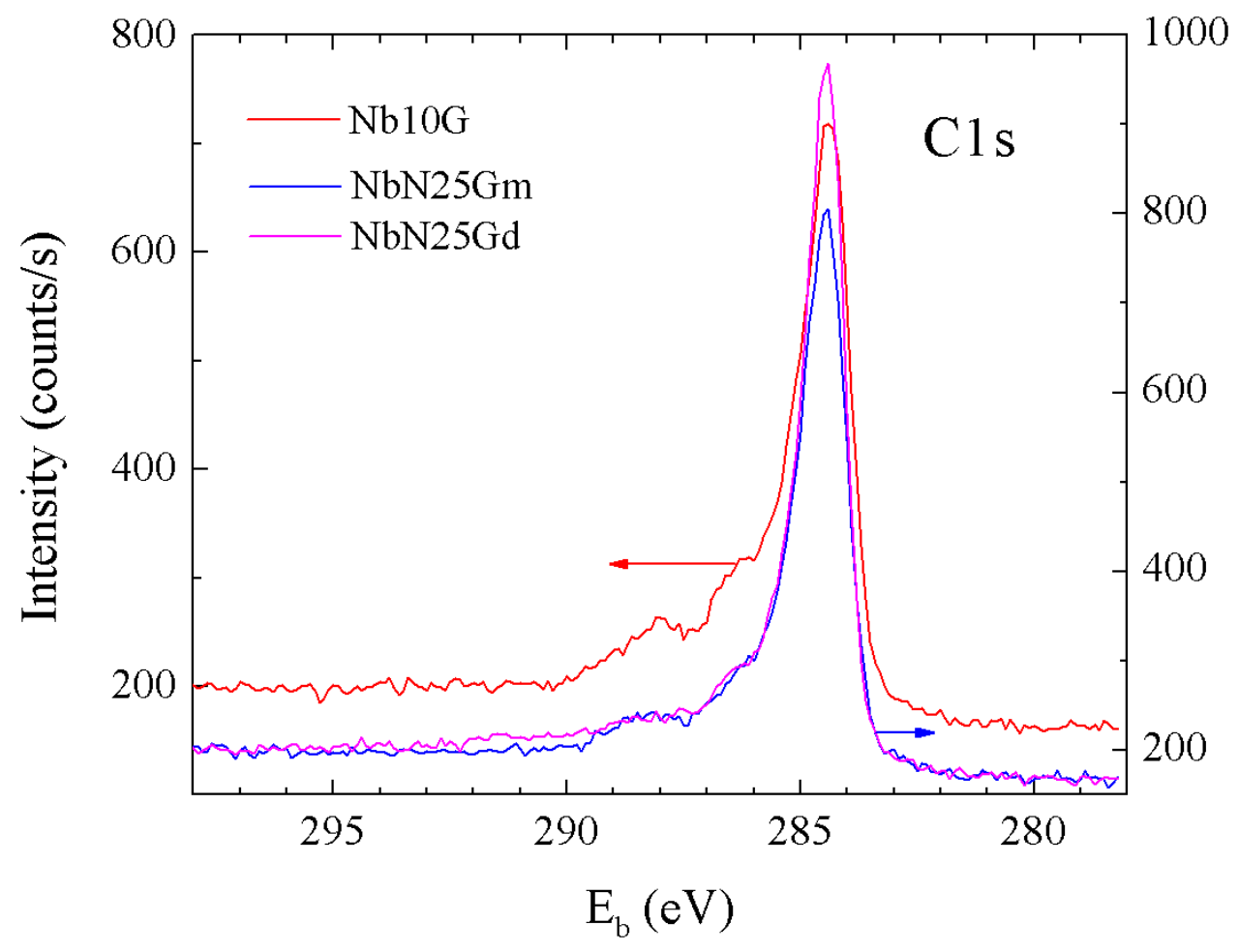

Figure C.3. HR XPS C 1s core level spectra of the samples Nb10G (red, left axis), $\mathrm{NbN25Gm}$ (blue, right axis), and $\mathrm{NbN25Gd}$ (brown, right axis).

The HR XPS Nb 3d core level spectrum shown in Fig. C.4(a) for the sample Nb10 reveals the presence of multiple oxidation states of $\mathrm{Nb}$ associated to different compounds. The standard values of the electron binding energy of the elements in different chemical environments were taken as a reference from the NIST X-ray Photoelectron Spectroscopy Database [74]. The experimental data could be deconvoluted into the ten peaks shown in Fig. C.4(a), which are related to $\mathrm{Nb}$ $\left(E_{\mathrm{b}}=202.1 \mathrm{eV}\right)$, to the niobium oxides, $\mathrm{NbO},\left(E_{\mathrm{b}}=202.9 \mathrm{eV}, 204.8 \mathrm{eV}\right), \mathrm{NbO}_{2}$, $\left(E_{\mathrm{b}}=205.9 \mathrm{eV}, 206.1 \mathrm{eV}, 208.7 \mathrm{eV}\right.$ ) and to the most stable (its band gap is $3.5 \mathrm{eV}$ ) niobium pentoxide, $\mathrm{Nb}_{2} \mathrm{O}_{5},\left(E_{\mathrm{b}}=207.4 \mathrm{eV}, 207.6 \mathrm{eV}, 210.2 \mathrm{eV}, 210.2 \mathrm{eV}\right)[74,75]$. The envelope of the fitted peaks (red line) corresponds to the experimental curve, which shows that sample Nb10 is in fact a Niobium/Niobium oxide bilayer. 
The HR XPS Nb 3d core level spectrum for Nb10G is shown in Fig. C.4(b). The main niobium metallic peak, $\mathrm{Nb} 3 \mathrm{~d}_{5 / 2}$ at $E_{\mathrm{b}}=202.1 \mathrm{eV}$ is still present, but its amplitude is smaller respect to the previous spectrum. Moreover, as demonstrated by the deconvolution of the experimental data, all the peaks of the niobium oxides [indicated by the same colors as in Fig. C.4(a)] are still present in the spectrum. Again, the envelope line plotted taking into account all the fitted peaks, perfectly describes the experimental data. Therefore, the sample Nb10Gm is a Niobium/Niobium oxide/Graphene trilayer.

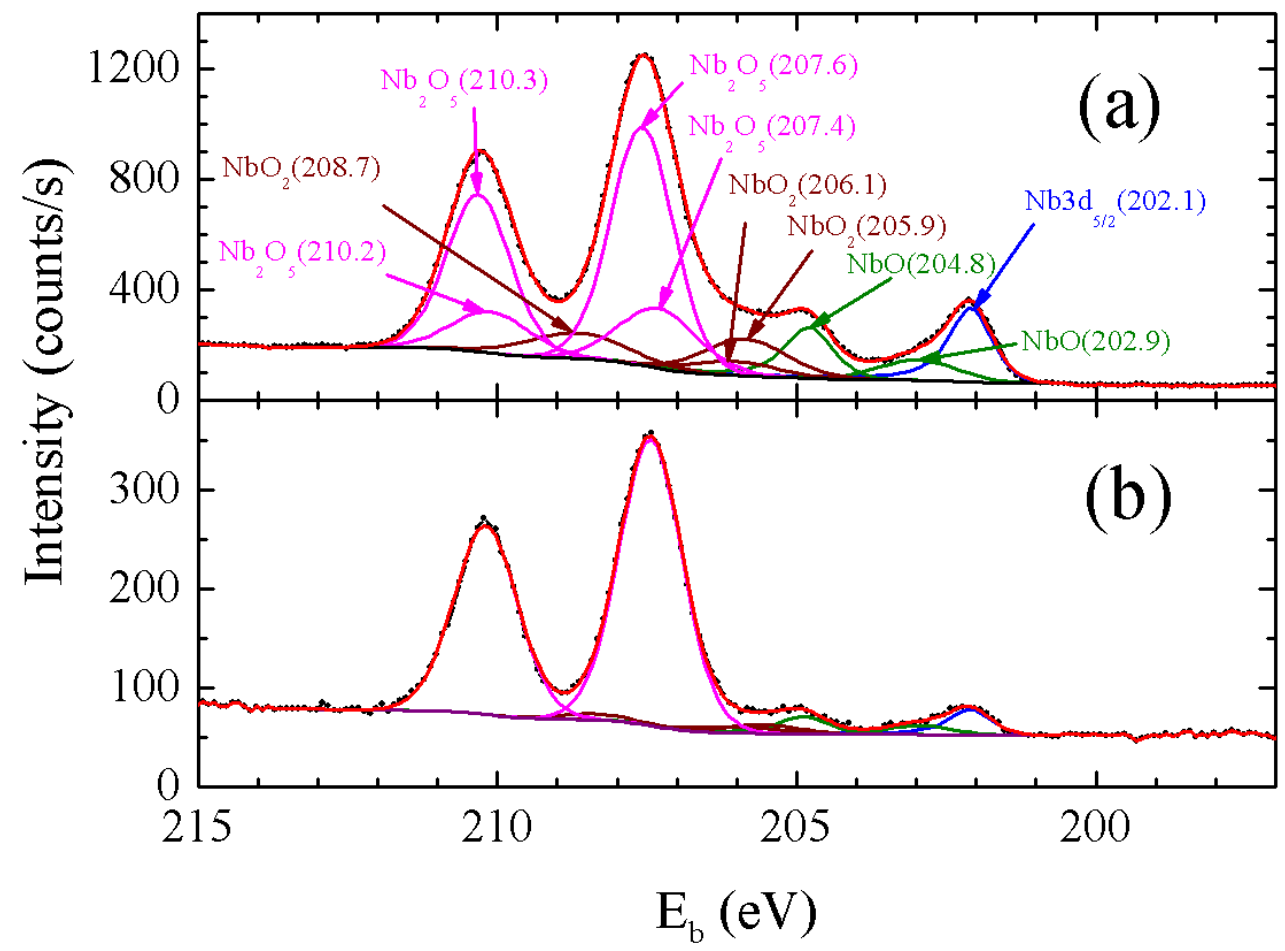

Figure C.4. HR XPS Nb 3d core level spectra (black points) of Nb10 (a) and Nb10G (b) after deconvolution (color lines). Arrows indicate the various states of $\mathrm{Nb}$. Numbers in brackets represent the binding energy in $\mathrm{eV}$. The colors in (a) and (b) are the same for the same states of $\mathrm{Nb}$. The red line is the envelope of the fitted peaks.

The HR XPS Nb 3d core level spectrum for NbN25 is shown in Fig. C.5(a). The analysis of the spectrum reveals the absence of the $\mathrm{Nb}$ peak and the presence of a strong $\mathrm{NbN}$ peak at $E_{\mathrm{b}}=204.1 \mathrm{eV}$. Moreover, the niobium pentoxide dominates and, from the slight asymmetry of the $\mathrm{NbN}$ peak, it follows that the sample contains also niobium oxynitride $(\mathrm{NbNO})\left(E_{\mathrm{b}}=205.3 \mathrm{eV}\right)$. The data confirm what 
already known from the literature for sputtered $\mathrm{NbN}$ films in which metallic $\mathrm{NbN}$ grains are firstly surrounded by $\mathrm{NbNO}$ and then by the more stable $\mathrm{Nb}_{2} \mathrm{O}_{5}$ layers [76]. This means that the $\mathrm{NbN} 25$ film is of complicated nature because the outermost layer is formed by a stable $\mathrm{Nb}_{2} \mathrm{O}_{5}$ followed by a dielectric oxynitride $\mathrm{NbNO}$, that is in turn adjacent to the metallic $\mathrm{NbN}$ [76]. The presence of the latter is indirectly confirmed by the HR XPS Nb 3d core level spectra measured for the samples NbN25Gm [Fig. C.5(b)] and NbN25Gd [Fig. C.5(c)]. Indeed, while the $\mathrm{Nb}_{2} \mathrm{O}_{5}$ peak becomes dominant, the peaks associated to the NbNO becomes smaller. This means that niobium oxynitride is moved further away from the surface of the sample.

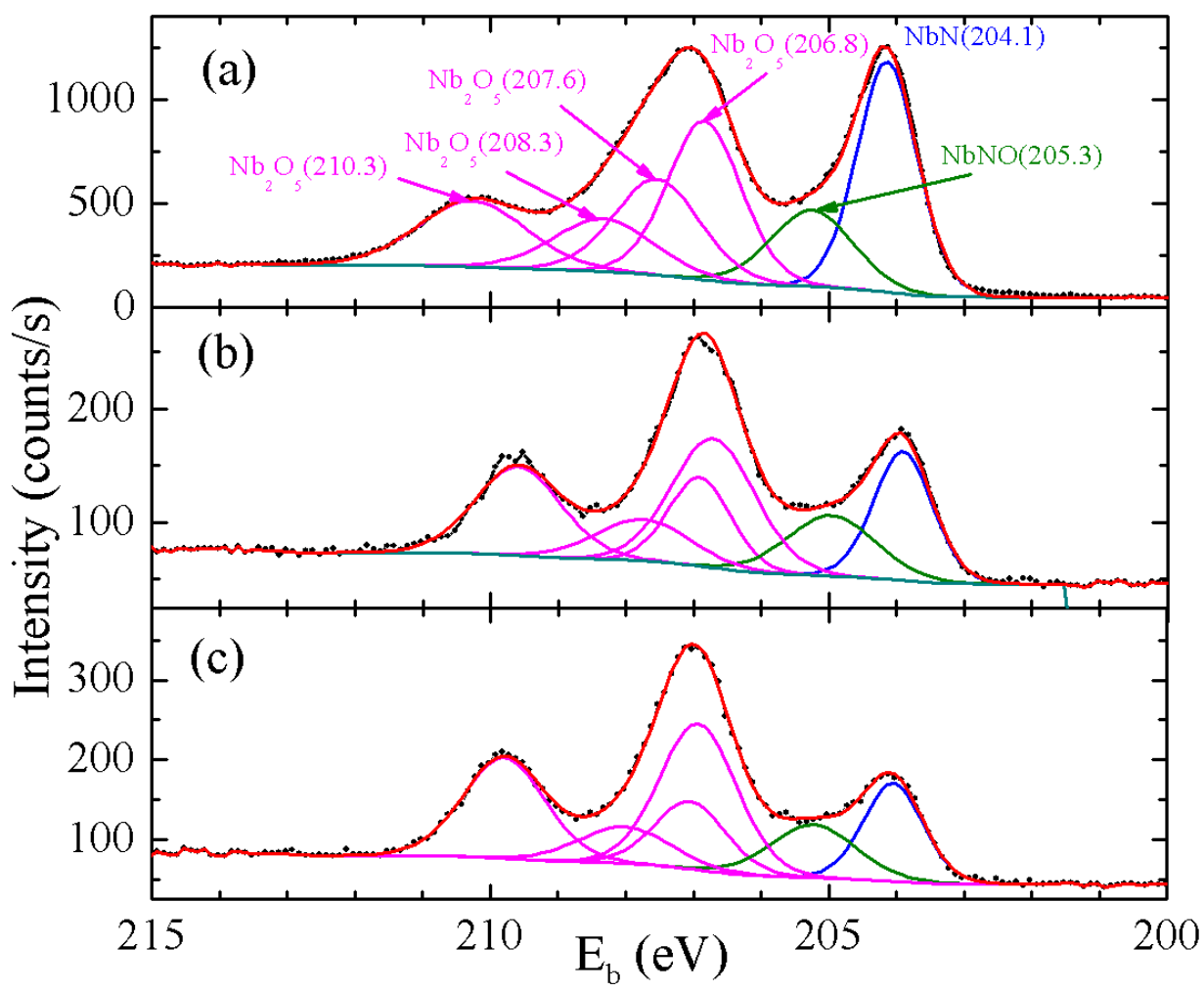

Figure C.5. HR XPS Nb3d core level spectra of (a) NbN25, (b) NbN25Gm and (c) NbN25Gd. Arrows indicate the different states of Nb. Numbers in brackets represent the binding energy in $\mathrm{eV}$. The colors in the panels $(a),(b)$ and $(c)$ are the same for the same states of niobium. The red line corresponds to the envelope of the fitted peaks. 


\section{References}

1. G. M. Eliashberg, Zh. Eksp. Teor. Fiz. 38, 966 (1960) [Sov. Phys. JETP 11, 696 (1960].

2. G. M. Eliashberg, Zh. Eksp. Teor. Fiz. 39, 1437 (1961) [Sov. Phys. JETP 12, 1000 (1961].

3. J. C. Swihart, D. J. Scalapino, and Y. Wada, Phys. Rev. Lett. 14, 106 (1965).

4. W. L. McMillan, Phys. Rev. 167, 331 (1968).

5. W. L. McMillan and J.M. Rowell, Phys. Rev. Lett. 14, 108 (1965).

6. J. W. Garland, K. H. Bennemann, and F. M. Mueller, Phys. Rev. Lett. 21, 1315 (1968).

7. P. B. Allen and R. C. Dynes, Phys. Rev. B 12, 905 (1975).

8. D. A. Kirzhnits, E. G. Maksimov, and D. I. Khomskii, J. Low Temp. Phys. 10, 79 (1973).

9. F. Giustino, Rev. Mod. Phys. 89, 015003 (2017).

10. O. V. Dolgov and E. G. Maksimov, Sov. Phys. Usp. 25, 688 (1982) [Usp. Fiz. Nauk 138, 95 (1982)].

11. E. G. Maksimov, D. Yu. Savrasov, and S. Yu. Savrasov, Phys. Usp. 40, 337 (1997) [Usp. Fiz. Nauk. 167, 353 (1997)].

12. P. Nozières and S. Schmitt-Rink, J. Low Temp. Phys. 59, 195 (1985).

13. J. M. Dickey and A. Paskin, Phys. Rev. Lett. 21, 1441 (1968).

14. R. E. Allen and F. W. de Wette, Phys. Rev. 187, 883 (1969).

15. G. Bergmann, Phys. Rev. B 3, 3797 (1971).

16. J. Noffsinger and M. L. Cohen, Phys. Rev. B 81, 214519 (2010).

17. E. Katzir, S. Yochelis, F. Zeides, N. Katz, Y. Kalcheim, O. Millo, G. Leitus, Y. Myasoedov, B. Ya. Shapiro, R. Naaman, and Y. Paltiel, Phys. Rev. Lett. 108, 107004 (2012).

18. E. Katzir, S. Yochelis, F. Zeides, N. Katz, S. Behrens, Y. Kalcheim, O. Millo, and Y. Paltiel. Europhys. Lett. 108, 37006 (2014).

19. H. Alpern, M. Periyasamy, J. Tannous, G. Jung, I. Zaytseva, A. Rosova, Š Chromik, V. Štrbik, M. Talacko, S. Yochelis, Y. Yacoby, O. Millo, and Y. Paltiel, J. Supercond. Nov. Magn. 33, 1941 (2020).

20. V. N. Kushnir, S. L. Prischepa, M. L. Della Rocca, M. Salvato, and C. Attanasio, Phys. Rev. B 68, 212505 (2003). 
21. V. N. Kushnir, S. L. Prischepa, C. Cirillo, M. L. Della Rocca, A. Angrisani Armenio, L. Maritato, M. Salvato, and C. Attanasio, Eur. Phys. J. B 41, 439 (2004).

22. S. L. Prischepa, C. Cirillo, V. N. Kushnir, E. A. Ilyina, M. Salvato, and C. Attanasio, Phys. Rev. B 72, 024535 (2005).

23. V. N. Kushnir, S. L. Prischepa, C. Cirillo, and C. Attanasio, Eur. Phys. J. B 52, 9 (2006).

24. V. N. Kushnir, E. A. Ilyina, S. L. Prischepa, C. Cirillo, and C. Attanasio, Superlatt. Microstruc. 43, 86 (2008).

25. E. L. Wolf. Principles of Electronic Tunneling Spectroscopy. Oxford: Oxford University Press. 1985.

26. E. L. Wolf and G. B. Arnold, Phys. Rep. 91, 31 (1982).

27. J. Bostock, W. N. Cheung, K. H. Lo, V. Diadiuk, and M. L. A. MacVicar, Phys. Rev. Lett. 36, 603 (1976).

28. K. Gärtner and A. Halm, Z. Naturforsch. 31a, 361 (1976).

29. J. Bostock and M. L. A. MacVicar, Phys. Lett. 71A, 373 (1979).

30. L. Y. L. Shen. In: Superconductivity in $d$ - and f-band Metals. AIP conf. proc. No.4 (American Institute of Physics, New York, 1972) pp. 31-44.

31. B. Robinson and T.H. Geballe In: Rochester Conference on Superconductivity in $d$ - and f-band Metals. (Plenum Press, New York, 1976) pp. 381-389.

32. A. V. Pogrebnyakov, J. M. Redwing, S. Raghavan, V. Vaithyanathan, D. G. Schlom, S. Y. Xu, Qi Li, D. A. Tenne, A. Soukiassian, X. X. Xi, M. D. Johannes, D. Kasinathan, W. E. Pickett, J. S. Wu, and J. C. H. Spence, Phys. Rev. Lett. 93, 147006 (2004).

33. J.C. Zheng and Y.M. Zhu, Phys. Rev. B 73, 024509 (2006).

34. W. X. Li, X. Xu, K. S. B. De Silva, F. X. Xiang, and S. X. Dou, IEEE Trans. Appl. Supercond. 23, 7000104 (2013).

35. A. Balandin, S. Ghosh, W. Z. Bao, I. Calizo, D. Teweldebrhan, F. Miao, and C. N. Lau, Nano Lett. 8, 902 (2008).

36. S. S. Chen, Q. Z. Wu, C. Mishra, J. Y. Kang, H. J. Zhang, K. J. Cho, W. W. Cai, A. A. Balandin, and R. S. Ruoff, Nature Mater. 11, 203 (2012).

37. J. H. Seol, I. Jo, A. L. Moore, L. Lindsay, Z. H. Aitken, M. T. Pettes, X. S. Li, Z. Yao, R. Huang, D. Broido, N. Mingo, R. S. Ruoff, and L. Shi, Science 328, 213 (2010). 
38. P. L. McEuen, X. D. Xu, N. M. Gabor, J. S. Alden, and A. M. van der Zande, Nano Lett. 10, 562 (2010).

39. M. H. Bae, Z. Y. Ong, D. Estrada, and E. Pop, Nano Lett. 10, 4787 (2010).

40. D. Liao, J. Z. Wu, X. R. Wang, K. Tahy, D. Jena, H. J. Dai, and E. Pop, Phys. Rev. Lett. 106, 256801 (2011).

41. T. H. Yu, C. W. Liang, C. Kim, E. S. Song, and B. Yu, IEEE Electr. Dev. Lett. 32, 1110 (2011).

42. Q. Shao, G. Liu, D. Teweldebrhan, and A. A. Balandin, Appl. Phys. Lett. 92, 202108 (2008).

43. Y. Wang, Z. Ni, T. Yu, Z. X. Shen, H. Wang, Y. Wu, W. Chen, and A. T. Shen Wee, J. Phys. Chem. C 112, 10637 (2008).

44. O. Frank, J. Vejpravova, V. Holy, L. Kavan, and M. Kalbac, Carbon 68, 440 (2014).

45. I. Calizo, S. Ghosh, W. Bao, F. Miao, C. N. Lau, and A. A. Balandin, Solid State Commun. 149, 1132 (2009).

46. E. A. Kolesov, M. S. Tivanov, O. V. Korolik, A. M. Saad, and I. V. Komissarov, Carbon 111, 587 (2017).

47. L. Chen and S. Kumar, J. Appl. Phys. 112, 043501 (2012).

48. C. H. Lui and T. F. Heinz, Phys. Rev. B 87, 121404(R) (2013).

49. L. Liang, J. Zhang, B. G. Sumpter, Q.-H. Tan, P.-H. Tan, and V. Meunier, ACS Nano 11, 11777 (2017).

50. C. H. Lui, L. M. Malard, S. H. Kim, G. Lantz, F. E. Laverge, R. Saito, and T.F. Heinz, Nano Lett. 12, 5539 (2012).

51. R. J. Nemanich, G. Lucovsky and S. A. Solin, Solid State Commun. 23, 117 (1977).

52. P. H. Tan, W. P. Han, W. J. Zhao, Z. H. Wu, K. Chang, H. Wang, Y. F. Wang, N. Bonini, N. Marzari, N. Pugno, G. Savini, A. Lombardo, and A. C. Ferrari, Nature Mater. 11, 294 (2012).

53. I. Cocemasov, D. L. Nika, and A. A. Balandin, Phys. Rev. B 88, 035428 (2013).

54. F. Wu, A. H. McDonald, and I. Martin, Phys. Rev. Lett. 121, 257001 (2018).

55. B. Lian, Z. Wang, and B. A. Bernevig, Phys. Rev. Lett. 122, 257002 (2019).

56. M. Trezza, C. Cirillo, P. Sabatino, G. Carapella, S. L. Prischepa, and C. Attanasio, Appl. Phys. Lett. 103, 252601 (2013). 
57. L. B. L. G. Pinheiro, M. Caputo, C. Cirillo, C. Attanasio, T. H. Johansen, W.A. Ortiz, A.V. Silhanek, and M. Motta, Low Temp. Phys. 46, 365 (2020) [Fiz. Nizk. Temp. 46, 441 (2020)].

58. I. V. Komissarov, N. G. Kovalchuk, V. A. Labunov, K. V. Girel, O. V. Korolik, M. S. Tivanov, A. Lazauskas, M. Andrulevičius, T. Tamulevičius, V. Grigaliūnas, Š. Meškinis, S. Tamulevičius, and S. L. Prischepa, Beilstein J. Nanotechnol. 8, 145 (2017).

59. I. V. Komissarov, N. G. Kovalchuk, E. A. Kolesov, M. S. Tivanov, O. V. Korolik, A. V. Mazanik, Yu. P. Shaman, A. S. Basaev, V. A. Labunov, S. L. Prischepa, N. I. Kargin, R. V. Ryzhuk, and S. A. Shostachenko, Phys. Proc. 72, 450 (2015).

60. J. Draskovic, T. R. Lemberger, B. Peters, F. Yang, J. Ku, A. Bezryadin, and S. Wang, Phys. Rev. B 88, 134516 (2013).

61. N. W. Ahscroft and N. D. Mermin. Solid State Physics. Brooks/Cole, 1976.

62. F. Marsiglio and J. P. Carbotte. Electron-Phonon Superconductivity. P. 73162 in K.H. Bennemann, J.B. Ketterson (eds.), Superconductivity, Vol. 1: Conventional and Unconventional Superconductors. Springer, Verlag Berlin Heidelberg 2008.

63. K. R. Babu and G.-Y. Guo, Phys. Rev. B 99, 104508 (2019).

64. R. Jani, N. E. Brener, and J. Callaway, Phys. Rev. B. 38, 9425 (1988).

65. C. Ferrari, J. C. Meyer, V. Scardaci, C. Casiraghi, M. Lazzeri, F. Mauri, S. Piscanec, D. Jiang, K. S. Novoselov, S. Roth, and A. K. Geim, Phys. Rev. Lett. 97, 187401 (2006).

66. J. E. Lee, G. Ahn, J. Shim, Y. S. Lee, and S. Ryu, Nat. Commun. 3, 1024 (2012).

67. E. Koo and S.-Y. Ju, Carbon 86, 318 (2015).

68. N. G. Kovalchuk, K. A. Nigerish, M. M. Mikhalik, N. I. Kargin, I. V. Komissarov, and S. L. Prischepa, J. Appl. Spectrosc. 84, 995 (2018).

69. S. Kim and S. Ryu, Carbon 100, 283 (2016).

70. V. G. Bayev, J. A. Fedotova, J. V. Kasiuk, S. A. Vorobyova, A. A. Sohor, I. V. Komissarov, N. G. Kovalchuk, S. L. Prischepa, N. I. Kargin, M. Andrulevičius, J. Przewoznik, Cz. Kapusta, O. A. Ivashkevich, S. I. Tyutyunnikov, N. N. Kolobylina, and P. V. Guryeva, Appl. Surf. Sci. 440, 1252 (2018). 
71. F. Moulder, W. F. Stickle, P. E. Sobol, and K. D. Bomben. Handbook of XRay Photoelectron Spectroscopy, Perkin-Elmer 1992.

72. K. L. Smith and K. M. Black, J. Vac. Sci. Technol. A2, 744 (1984).

73. T. Susi, T. Pichler, and P. Ayala, Beilstein J. Nanotechnol. 6, 177(2015).

74. http://srdata.nist.gov/xps/selEnergyType.aspx.

75. Z. Yang, X. Lu, W. Tan, J. Zhao, D. Yang, Y. Yang, Y. He, and K. Zhou, Appl. Surf. Sci. 439, 1119 (2018).

76. A. Darlinski and J. Halbritter, Surface and Interface Analysis 10, 223 (1987). 